\title{
A MODEL FOR THE WAVEFORM BEHAVIOR OF ACCRETING MILLISECOND X-RAY PULSARS: NEARLY ALIGNED MAGNETIC FIELDS AND MOVING EMISSION REGIONS
}

\author{
Frederick K. Lamb ${ }^{1,2}$, Stratos Boutloukos ${ }^{1,3}$, Sandor Van Wassenhove ${ }^{1}$, Robert T. Chamberlain ${ }^{1}$, Ka Ho Lo ${ }^{1}$, \\ Alexander Clare ${ }^{1}$, Wenfei Yu ${ }^{1}$, and M. Coleman Miller ${ }^{3}$ \\ ${ }^{1}$ Center for Theoretical Astrophysics and Department of Physics, University of Illinois at Urbana-Champaign, 1110 West Green Street, \\ Urbana, IL 61801-3080, USA; fkl@illinois.edu \\ 2 Department of Astronomy, University of Illinois at Urbana-Champaign, 1002 West Green Street, Urbana, IL 61801-3074, USA \\ ${ }^{3}$ Department of Astronomy and Maryland Astronomy Center for Theory and Computation, University of Maryland, College Park, MD 20742-2421, USA \\ Received 2008 August 31; accepted 2009 October 5; published 2009 October 30
}

\begin{abstract}
We investigate further a model of the accreting millisecond X-ray pulsars we proposed earlier. In this model, the $\mathrm{X}$-ray-emitting regions of these pulsars are near their spin axes but move. This is to be expected if the magnetic poles of these stars are close to their spin axes, so that accreting gas is channeled there. As the accretion rate and the structure of the inner disk vary, gas is channeled along different field lines to different locations on the stellar surface, causing the X-ray-emitting areas to move. We show that this "nearly aligned moving spot model" can explain many properties of the accreting millisecond X-ray pulsars, including their generally low oscillation amplitudes and nearly sinusoidal waveforms; the variability of their pulse amplitudes, shapes, and phases; the correlations in this variability; and the similarity of the accretion- and nuclear-powered pulse shapes and phases in some. It may also explain why accretion-powered millisecond pulsars are difficult to detect, why some are intermittent, and why all detected so far are transients. This model can be tested by comparing with observations the waveform changes it predicts, including the changes with accretion rate.
\end{abstract}

Key words: pulsars: general - stars: neutron - stars: rotation - X-rays: bursts - X-rays: stars

\section{INTRODUCTION}

Highly periodic millisecond X-ray oscillations have been detected with high confidence in 22 accreting neutron stars in low-mass X-ray binary systems (LMXBs), using the Rossi $X$ ray Timing Explorer (RXTE) satellite (see Lamb \& Boutloukos 2008). We refer to these stars as accreting millisecond $X$-ray pulsars (AMXPs). Accretion-powered millisecond oscillations have so far been detected in 10 AMXPs. They are always observable in seven AMXPs, but are only intermittently detected in three others. Nuclear-powered millisecond oscillations have been detected with high confidence during thermonuclear Xray bursts in 16 AMXPs. Persistent accretion-powered millisecond oscillations have been detected in two AMXPs that produce nuclear-powered millisecond oscillations; intermittent accretion-powered millisecond oscillations have been detected in two others.

The AMXPs have several important properties:

Low oscillation amplitudes. The fractional amplitudes of the accretion-powered oscillations of most AMXPs are often only $\sim 1 \%-2 \% .{ }^{4}$ Persistent accretion-powered oscillations with amplitudes $\lesssim 1 \%$ are often detected with high confidence in IGR J00291+5934 (Galloway et al. 2005; Patruno 2008) and XTE J1751-305 (Markwardt et al. 2002; Patruno 2008). Persistent accretion-powered oscillations with amplitudes as low as $2 \%$ are regularly seen in XTE J1807-294 (Zhang et al. 2006; Chou et al. 2008; Patruno 2008), XTE J0929-314 (Galloway et al. 2002), and XTE J1814-338 (Chung et al. 2008; Patruno 2008). The

\footnotetext{
4 We characterize the strengths of oscillations by their rms amplitudes because the rms amplitude can be defined for any waveform, is usually relatively stable, and is closely related to the power. We convert reported semi-amplitudes of purely sinusoidal oscillations or Fourier components to rms amplitudes by dividing by $\sqrt{2}$.
}

amplitude of the accretion-powered oscillation seen in SWIFT 1756.9-2508 was $\sim 6 \%$ (Krimm et al. 2007). The intermittent accretion-powered oscillations detected in SAX J1748.9-2021 (Gavriil et al. 2007; Altamirano et al. 2008; Patruno 2008), HETE J1900.1-2455 (Galloway et al. 2007), and Aql X-1 (Casella et al. 2008) have amplitudes $\sim 0.5 \%-3 \%$.

Nearly sinusoidal waveforms. The waveforms (light curves) of the accretion-powered oscillations of most AMXPs are nearly sinusoidal (see Wijnands 2006 and the references in the preceding paragraph). The amplitude of the first harmonic (fundamental) component is usually $\gtrsim 10$ times the amplitude of the second harmonic (first overtone) component, although the ratio can be as small as $\sim 3.5$, as is sometimes the case in XTE J1807-294 (Zhang et al. 2006), or even $\gtrsim 1$, as is sometimes the case in SAX J1808.4-3658 (see, e.g., Hartman et al. 2008).

Highly variable oscillation amplitudes. The fractional amplitudes of the accretion-powered oscillations of most AMXPs vary in time by factors ranging from $\sim 2$ to $\sim 10$. Observed fractional amplitudes vary from $0.7 \%$ to $1.7 \%$ in SAX J1748.9-2021, from $0.7 \%$ to $3.7 \%$ in XTE J1751-305, from 3\% to 7\% in XTE J0929-314, from $1 \%$ to $9 \%$ in IGR J00291+5934, from $2 \%$ to $11 \%$ in XTE J1814-338, from $1 \%$ to $14 \%$ in XTE J1808-338, and from 2\% to 19\% in XTE J1807-294 (see the references above).

Highly variable pulse phases. The phases of accretionpowered pulses have been seen to vary rapidly by as much as $\sim 0.3$ cycles in several AMXPs, including SAX J1808.4-3658 (Morgan et al. 2003; Hartman et al. 2008) and XTE J1807-294 (Markwardt 2004). Wild changes in the apparent pulse frequency have been observed with both signs at the same accretion rate in XTE J1807-294 (see Markwardt 2004). If interpreted as caused by changes in the stellar spin rate, these phase variations would be more than a factor of 10 larger than expected for the largest accretion torques and smallest inertial moments thought 
possible for these systems (see Ghosh \& Lamb 1979b; Lattimer \& Prakash 2001).

Undetected accretion-powered oscillations. More than 80 accreting neutron stars in LMXBs are known (Chakrabarty 2005; Liu et al. 2007), but accretion-powered millisecond X-ray oscillations have so far been detected in only 10 of them. Accretionpowered oscillations have not yet been detected even in 13 AMXPs that produce periodic nuclear-powered millisecond oscillations, indicating that they have millisecond spin periods (Lamb \& Boutloukos 2008); eight of these also produce kilohertz quasi-periodic oscillations (QPOs) with frequency separations that indicate that they not only have millisecond spin periods but also have dynamically important magnetic fields (Boutloukos \& Lamb 2008).

Intermittent accretion-powered oscillations. Accretionpowered millisecond X-ray pulsations have been detected only occasionally in SAX J1748.9-2021 (Gavriil et al. 2007; Altamirano et al. 2008; Patruno 2008), HETE J1900.1-2455 (Galloway et al. 2007), and Aql X-1 (Casella et al. 2008). When oscillations are not detected, the upper limits are typically $\lesssim 0.5 \%$.

Correlated pulse arrival times and amplitudes. The phase residuals of the accretion-powered pulses of several AMXPs appear to be anti-correlated with their fractional amplitudes, at least over some of the amplitude ranges observed. AMXPs that show this type of behavior include XTE J1807-294 and XTE J1814-338 (Patruno 2008).

Similar accretion- and nuclear-powered pulses. The shapes and phases of the nuclear-powered X-ray pulses of the AMXPs SAX J1808.4-3658 (Chakrabarty et al. 2003) and XTE J1814-338 (Strohmayer et al. 2003) are very similar to the shapes and phases of their accretion-powered X-ray pulses.

Concentration in transient systems of AMXPs with accretionpowered oscillations. The AMXPs in which accretion-powered oscillations have been detected tend to be found in binary systems that have outbursts lasting about a month (but see Galloway et al. 2008) separated by quiescent intervals lasting years (Chakrabarty 2005; Riggio et al. 2008). The accretion rates of these neutron stars are very low.

In this paper, we investigate further the "nearly aligned moving spot" model of AMXP X-ray emission that we proposed previously (Lamb et al. 2006, 2007, 2008). This model has three main features:

1. The strongest poles of the magnetic fields of neutron stars with millisecond spin periods are located near-and sometimes very near-the stellar spin axis. This behavior is expected for several magnetic field evolution mechanisms.

2. The star's magnetic field channels accreting gas close to its spin axis, creating X-ray-emitting areas there and depositing nuclear fuel there. ${ }^{5}$

3. The X-ray-emitting areas on the stellar surface move, as changes in the accretion rate and the structure of the inner disk cause accreting gas to be channeled along different field lines to different locations on the stellar surface. (The magnetic field of the neutron star is fixed in the stellar crust on the timescales relevant to the phenomena considered here.)

\footnotetext{
5 Muno et al. (2002) considered a single bright spot near the spin axis as well as a uniformly bright hemisphere and antipodal spots near the spin equator as possible reasons for the nearly sinusoidal waveforms of some X-ray burst oscillations, but did not consider accretion-powered oscillations or other consequences of emission from near the spin axis.
}

These features provide the basic ingredients needed to understand the AMXP properties discussed above. This is the subject of the sections that follow. As a guide to these sections, we summarize our results here.

1. Emission from near the spin axis naturally produces weak modulation, regardless of the viewing direction. The reason is that uniform emission from a spot centered on the spin axis is axisymmetric about the spin axis and therefore produces no modulation. Emission from a spot close to the spin axis has only a small asymmetry and therefore produces only weak modulation.

2. Emission from near the spin axis also naturally produces a nearly sinusoidal waveform, because the asymmetry of the emission is weak and broad.

3. If the emitting area is close to the spin axis, even a small movement in latitude can change the oscillation amplitude by a substantial factor.

4. If the emitting area is close to the spin axis, movement in the longitudinal direction by a small distance can change the phase of the oscillation by a large amount.

Changes in the latitude and longitude of the emitting area are expected on timescales at least as short as the $\sim 0.1 \mathrm{~ms}$ dynamical time at the stellar surface and as long as the $\sim 10$ day timescale of the variations observed in the mass accretion rate.

5. If the emitting area is very close to the spin axis and remains there, the oscillation amplitude may be so low that it is undetectable. The effects of rapid changes in the position of the emitting area-possibly in combination with other effects, such as reduction of the modulation fraction by scattering in circumstellar gas-may also play a role in reducing the detectability of accretion-powered oscillations in neutron stars with millisecond spin periods. These effects may explain the fact that accretion-powered $\mathrm{X}$-ray oscillations have not yet been detected in many accreting neutron stars that are thought to have millisecond spin periods and dynamically important magnetic fields.

6. If the emitting area is very close to the spin axis, a small change in the accretion flow can suddenly channel gas farther from the spin axis, causing the emitting area to move away from the axis. This can make a previously undetectable oscillation become detectable. Temporary motion of the emitting area away from the spin axis may explain the intermittent accretion-powered oscillations of some AMXPs (Lamb et al. 2009).

7. If the pulse amplitude and phase variations observed in AMXPs are caused by motion of the emitting area, they should be correlated. In particular, the pulse phase should be much more scattered when the pulse amplitude is very low. The reason is that changes in the longitudinal position of the emitting area by a given distance produce much larger phase changes when the emitting area is very close to the spin axis, which is also when the oscillation amplitude is very low.

The observational consequences discussed so far depend only on features (2) and (3) of the model, i.e., that the accretion-powered X-ray emission of AMXPs comes from areas near their spin axes and that these areas move significantly on timescales of hours to days.

8. The picture of AMXP X-ray emission outlined here suggests that the shapes and phases of the nuclear- and accretion-powered pulses are similar to one another in some AMXPs because the nuclear- and accretion-powered X-ray 
emission comes from approximately the same area on the stellar surface. The reason for this is that in some cases, the mechanism that concentrates the magnetic flux of the accreting neutron star near its spin axis, as it is spun up, will naturally produce magnetic fields strong enough to confine accreting nuclear fuel near the magnetic poles at least partially, even though the dipole component of the magnetic field is weak.

9. The picture of neutron star magnetic field evolution and AMXP X-ray emission outlined here also suggests a possible explanation for why the AMXPs in which accretionpowered oscillations have been detected are in transient systems. If most neutron stars in LMXBs were spun up by accretion from a low spin rate to a high spin rate, their magnetic poles were forced very close to their spin axes, making accretion-powered oscillations difficult or impossible to detect. However, those stars that are now in compact transient systems now experience infrequent episodes of mass accretion, and the accretion rate is very low. By now they have been spun down from their maximum spin rates, a process that could force their magnetic poles away from their spin axes enough to produce detectable accretion-powered oscillations.

These last two observational consequences depend on feature (1) of the model, i.e., on how the magnetic fields of neutron stars evolve as they are spun up and down by accretion and electromagnetic torques.

In the remainder of this paper, we discuss in detail the features of the model and its observational implications. In Section 2, we outline our approach, discussing our modeling of X-ray emission from the stellar surface, our computational and the code verification methods, and the pulse profile representation we use.

We present our results in Sections 3 and 4. These results are based on our computations of several hundred million waveforms for different emitting regions, beaming patterns, stellar models, and viewing directions. In Section 3, we consider the shape and amplitude of X-ray pulses as a function of the size and inclination of the emitting areas, the compactness of the star, and the stellar spin rate. In Section 4, we consider the changes in the pulse amplitude and phase produced by various motions of the emitting regions on the stellar surface and explore the origins of correlated changes in the pulse amplitude and phase and the effects of rapid movement of the emitting areas. We also discuss why oscillations have not yet been detected in many accreting neutron stars in LMXBs and why accretion-powered oscillations are detected only intermittently in some AMXPs.

In Section 5, we summarize the results of our model calculations. We also discuss how the magnetic poles of most AMXPs can be forced close to their spin axes, how such mechanisms may explain why the AMXPs that produce accretion-powered millisecond oscillations are transient pulsars, the consistency of the model with the observed properties of rotation-powered millisecond pulsars, and possible observational tests of the model discussed here.

Further results of our investigation of the present model will be presented elsewhere (S. Boutloukos et al. 2009, in preparation).

\section{X-RAY WAVEFORM MODELING}

\subsection{Modeling the $X$-ray Emission}

In the radiating spot model of AMXP X-ray emission, the waveform seen by a distant observer depends on the sizes, shapes, and positions of the emitting regions on the stellar surface; the beaming pattern of the radiation; the compactness, radius, and spin rate of the star; and the direction from which the star is observed. The properties of the $\mathrm{X}$-ray-emitting regions are determined by the strength and geometry of the star's magnetic field, the locations where plasma from the accretion disk enters the magnetosphere, the extent to which the accreting plasma becomes threaded and channeled by the stellar magnetic field, and the resulting plasma flow pattern onto the stellar surface.

In principle, accreting plasma can reach the stellar surface in two basic ways: (1) by becoming threaded by the stellar magnetic field and then guided along stellar field lines to the vicinity of a stellar magnetic pole (Lamb et al. 1973; Basko \& Sunyaev 1975; Elsner \& Lamb 1976; Ghosh et al. 1977; Ghosh \& Lamb 1979a, 1979b) or (2) by penetrating between lines of the stellar magnetic field via the magnetic version of the Rayleigh-Taylor instability (Lamb 1975a, 1975b; Elsner \& Lamb 1976, 1977; Arons \& Lea 1976; Lamb 1977) and then spiraling inward to the stellar surface.

If a centered dipole component is the strongest component of the star's magnetic field, plasma in the accretion disk that becomes threaded and then channeled to the vicinity of a magnetic pole is expected to impact the star in a partial or complete annulus around the pole, producing a crescent- or ring-shaped emitting area near the pole. If the axis of the dipole field is significantly tilted relative to the spin axis and the spin axis is aligned with the axis of the accretion disk, a crescentshaped emitting region is expected (see, e.g., Basko \& Sunyaev 1975, 1976; Ghosh et al. 1977; Daumerie et al. 1996; Miller 1996; Miller et al. 1998; Romanova et al. 2003). If instead the dipole axis is very close to the spin axis, as in the model of AMXP X-ray emission proposed here, the emitting region may completely encircle the spin axis (see, e.g., Ghosh \& Lamb 1979a, 1979b; Romanova et al. 2003).

The north and south magnetic poles of some AMXPs may be very close to the same spin pole, producing a very offcenter dipole moment orthogonal to the spin axis (see Chen \& Ruderman 1993; Chen et al. 1998; and Section 5.1). If so, accreting matter will be channeled close to the spin axis, but may be channeled preferentially toward one magnetic pole, producing an emitting region with approximately onefold symmetry about the spin axis, or about equally toward both poles, producing an emitting region with approximately twofold symmetry about the spin axis. In the first case, the first harmonic of the spin frequency is likely be the dominant harmonic in the X-ray waveform, whereas in the second case, the second harmonic is likely to dominate. Which case occurs will depend on the accretion flow through the inner disk. In either case, the X-ray emission will come from close to the spin axis.

The neutron stars that are AMXPs may well have even more complicated magnetic fields, with significant quadrupole and octopole components. Higher multipole components are likely to play a more important role in the AMXPs than in the classic strong-field accretion-powered pulsars, because the magnetic fields of the AMXPs are much weaker. As a result, accreting plasma can penetrate closer to the stellar surface, where the higher multipole moments of the star's magnetic field have a greater influence on the channeling of accreting plasma (Elsner \& Lamb 1976). In this case, plasma will still tend to be channeled toward regions on the surface where the magnetic field is strongest and will tend to impact the surface in rings or annuli, but the emission pattern may be spatially complex and vary rapidly in time (Long et al. 2008). 
Disk plasma that penetrates between lines of the stellar magnetic field will continue to drift inward as it loses its angular momentum, probably predominantly via its interaction with the star's magnetic field (Lamb \& Miller 2001). Cold plasma will remain in the disk plane and impact the star in an annulus where the disk plane intersects the stellar surface (Miller et al. 1998; Lamb \& Miller 2001). If some of the accreting plasma were to become hot, the forces exerted on it by the stellar magnetic field would tend to drive it toward the star's magnetic equator (Michel 1977), causing it to impact the stellar surface in an annulus around the star's magnetic equator. However, emission and inverse Compton scattering of radiation is likely to keep the accreting plasma cold (Elsner \& Lamb 1984), so that it remains in the disk plane as it drifts inward. Plasma that penetrates to the stellar surface via the magnetic Rayleigh-Taylor instability is likely to impact the stellar surface in rapidly fluctuating, irregular patterns (see Romanova et al. 2006, 2008).

Whether accreting plasma reaches the stellar surface predominantly via channeled flow along field lines or via unstable flow between field lines depends on the accretion rate and the spin frequency of the star (see Lamb 1989; Romanova et al. 2008; Kulkarni et al. 2008). Under some conditions, plasma may accrete in both ways simultaneously (see Miller et al. 1998; Romanova et al. 2008; Kulkarni et al. 2008).

The sizes, shapes, and locations of the emitting areas on the surface of an accreting magnetic neutron star and the properties of the emission from these areas are expected to change on timescales at least as short as the $\sim 1 \mathrm{~ms}$ dynamical timescale near the star. This expectation is supported by recent simulations of accretion onto weakly magnetic neutron stars (see Romanova et al. 2003, 2004, 2006; Long et al. 2008; Romanova et al. 2008; Kulkarni et al. 2008). However, changes in AMXP X-ray fluxes can be measured accurately using current instruments only by combining $\sim 100-1000 \mathrm{~s}$ of data and hence only variations in waveforms on timescales longer than this can be measured directly. Consequently, the emitting areas and beaming patterns that are relevant for comparisons with current observations of waveforms are the averages of the actual areas and beaming patterns over these relatively long times. The emitting areas and beaming patterns that we use in our computations should therefore be interpreted as averages of the actual areas and beaming patterns over these times.

We have computed the X-ray waveforms produced by emitting regions with various sizes, shapes, and positions, for several different X-ray-beaming patterns and a range of stellar masses, compactnesses, and spin rates. We find that in many cases these waveforms can be approximated by the waveforms generated by a circular, uniformly emitting spot located at the centroid of the emitting region. The main reason for this is that an observer sees half the star's surface at a time (or more, when gravitational light deflection is included), which diminishes the influence of the size and detailed shape of the emitting region on the waveform. Consequently, we focus here on the waveforms produced by uniformly emitting circular spots. We will discuss the waveforms produced by emitting areas with other shapes, such as rings or crescents, in a subsequent paper (S. Boutloukos et al. 2009 , in preparation).

In addition to studying the X-ray waveforms produced by emitting areas with fixed sizes, shapes, positions, and radiationbeaming patterns, we are also interested in the changes in waveforms produced by changes in the these properties of the emitting areas. The changes we investigate should be understood as occurring on the timescales $\gtrsim 100$ s that can be studied using current instruments. It is not yet possible to compute from first principles the accretion flows and X-ray emission of AMXPs on these timescales, so simplified models must be used. (The simulations referred to earlier follow the accretion flow for a few dozen spin periods or dynamical times, intervals that are orders of magnitude shorter than the intervals that are relevant).

In the following sections, we consider radiation from a single spot, from two antipodal spots, and from two spots in the same rotational hemisphere, near the star's spin axis. Although many uncertainties remain, recent magnetohydrodynamic simulations of accretion onto weakly magnetic neutron stars have found that gas impacts $1 \%-20 \%$ of the stellar surface (Romanova et al. 2004), equivalent to the areas of circular spots with angular radii of $10^{\circ}-53^{\circ}$. These radii are consistent with analytical estimates of the sizes of the emission regions of accreting neutron stars with weak magnetic fields (Miller et al. 1998; Psaltis \& Chakrabarty 1999). Consequently, we focus on spot sizes in this range.

An observer may see radiation from a single spot either because the accretion flow pattern strongly favors one pole of a dipolar stellar magnetic field over the other, or because the observer's view of one pole is blocked by the inner disk or by accreting plasma in the star's magnetosphere (see McCray \& Lamb 1976; Basko \& Sunyaev 1976). An observer may see radiation from two antipodal spots if emission from both magnetic poles is visible. Finally, an observer may see radiation from two spots near the same rotation pole if neutron vortex motion drives both of the star's dipolar magnetic poles toward the same rotation pole (see Chen et al. 1998).

To make it easier for the reader to compare cases, we usually report results for our "reference" star, which is a $1.4 M_{\odot}$ star with a radius of $5 M$ in units where $G=c=1(10.3 \mathrm{~km}$ for $M=1.4 M_{\odot}$ ), spinning at $400 \mathrm{~Hz}$ as measured at infinity, but we also discuss other stellar models. For the same reason, we usually consider spots with angular radii of $25^{\circ}$. This is not an important limitation, because the observed waveform depends only weakly on the size of the emitting spots, as discussed in Section 3.2. We describe how the results change if the spot is larger or smaller.

\subsection{Computing X-ray Waveforms}

The X-ray waveforms calculated here assume that radiation propagating from emitting areas on the stellar surface reaches the observer without interacting with any intervening matter. The bolometric X-ray waveforms that would be seen by a distant observer were calculated using the Schwarzschild plus Doppler $(\mathrm{S}+\mathrm{D})$ approximation introduced by Miller \& Lamb (1998). The S+D approximation treats exactly the special relativistic Doppler effects (such as aberrations and energy shifts) associated with the rotational motion of the stellar surface, but treats the star as spherical and uses the Schwarzschild spacetime to compute the general relativistic redshift, trace the propagation of light from the stellar surface to the observer, and calculate light travel-time effects. For the stars considered here, and indeed for any stars that do not both rotate rapidly and have very low compactness, the effects of stellar oblateness and frame dragging are minimal and are negligible compared to uncertainties in the X-ray emission (see Cadeau et al. 2007).

We describe the emission from the stellar surface using coordinates centered on the star. When considering emission from a single spot, we denote the angle between its centroid 
and the star's spin axis by $i_{s}$ and its azimuth in the stellar coordinate system by $\phi_{s}$. When considering emission from two spots, we somewhat arbitrarily identify one as the primary spot and the other as the secondary spot. We denote the inclination and azimuth of the centroid of the primary spot by $i_{s 1}$ and $\phi_{s 1}$ and the inclination and azimuth of the centroid of the secondary spot by $i_{s 2}$ and $\phi_{s 2}$. We denote the inclination of the observer relative to the stellar spin axis by $i$.

In computing the waveforms seen by distant observers, we use as our global coordinate system Schwarzschild coordinates $(r, \theta, \varphi, t)$ centered on the star with $\theta=0$ aligned with the star's spin axis and $\varphi=0$ in the plane containing the spin axis and the observer. We choose the zero of the Schwarzschild time coordinate $t$ so that a light pulse that propagates radially from a point on the stellar surface immediately below the observer (i.e., at $\theta=i$ and $\varphi=0$ ) arrives at the observer at $t=0$.

We carried out many calculations to test and verify the computer code used to obtain the results we report here. We determined that the code was giving sufficiently accurate results by varying the spatial and angular resolutions used. For most of the cases considered in this paper, the emitting spots were sampled by a grid of 250 points in latitude and 250 points in longitude, the radiation-beaming pattern was specified at $10^{4}$ angles, and the flux seen by a distant observer was computed at $10^{4}$ equally spaced values of the star's rotational phase. In some cases, finer grids were used.

We verified the code used here by comparing its results with analytical and numerical results for several test cases:

1. We tested our code's representation of emitting areas and ray tracing in flat space by comparing the results given by our code with exact analytical results for the absolute flux seen by an observer directly above uniform, isotropically emitting circular spots of various sizes. The numerical results agreed with the analytical results.

2. We tested our code's computation of special relativistic Doppler boosts, aberrations, and propagation-time effects in several ways. We compared the results given by our code with exact analytical results for the waveforms produced by emission in (a) a pencil beam normal to the surface and (b) a thin fan beam tangent to the surface of a rapidly rotating star. We also compared the results given by our code with analytical results for the waveforms produced by a small spot on the surface of a slowly rotating star in flat space emitting (a) isotropically and (b) in a beaming pattern representing Comptonized emission (see Poutanen $\&$ Gierliński 2003). The numerical results agreed with the analytical results.

3. We tested our code's computation of the general relativistic redshift and light deflection for nonrotating stars by (a) comparing the deflection of a fan beam tangent to the stellar surface given by our code for a variety of stellar compactnesses with the analytical expressions for the light deflection given by Pechenick et al. (1983) and Page (1995); (b) comparing the absolute flux given by our code for an observer directly above isotropically emitting uniform circular spots of various sizes with independent semi-analytical results for these cases; (c) comparing the symmetries of the waveform and the phase of the waveform maximum given by our code with exact analytical results for these quantities; and (d) comparing the shape of the waveforms given by our code with the shapes reported by Pechenick et al. (1983) and Strohmayer (1992). Our numerical results agreed satisfactorily with the comparison results in all cases. ${ }^{6}$

4. We tested our code's computation of the waveforms produced by emission from slowly rotating stars in general relativity by comparing the rms oscillation amplitudes it gives with the amplitudes given by the approximate analytical formulae of Viironen \& Poutanen (2004). Where the results of Viironen \& Poutanen are expected to be accurate, the two sets of rms amplitudes agreed to better than 1\%; in many cases the agreement was much better. We also compared the waveform given by our code for an isotropically emitting spot inclined $45^{\circ}$ from the spin axis of a $1.4 M_{\odot}$ star with a Schwarzschild coordinate radius of $5 M$ spinning at $600 \mathrm{~Hz}$ seen by an observer at an inclination of $45^{\circ}$ with the waveform reported by Poutanen \& Beloborodov (2006) for this case; the two waveforms agreed to better than $1 \%$.

Further details of these tests and comparisons will be given in a subsequent paper (S. Boutloukos et al. 2009, in preparation).

\subsection{Constructing Pulse Profiles}

The X-ray flux seen by a given observer will evolve continuously in time as the star rotates and the emission from the stellar surface changes, generating the observed waveform $W(t)$. As noted in Section 2.1, the accretion flow from the inner disk to the stellar surface is expected to vary on timescales at least as short as the $\sim 1$ ms dynamical timescale near the neutron star, which will cause the sizes, shapes, and positions of the emitting regions, and therefore the observed waveform, to vary on these timescales.

The sensitivity of current instruments is too low to measure the waveform of an AMXP on timescales as short as $1 \mathrm{~ms}$. However, nearly periodic waveforms with periods this short can be partially characterized by folding segments of flux data centered at a sequence of clock times $t_{i}$ (see, e.g., Hartman et al. 2008; Patruno 2008). If the data are folded with a period $P_{f}$ that is chosen to agree as closely as possible with the local, approximate repetition period $P\left(t_{i}\right)$ of the waveform, one can construct a time sequence of pulse profiles $W_{P}\left(\phi, t_{i}\right)$; here $\phi$ is the pulse phase over one cycle.

The pulse profiles $W_{P}\left(\phi, t_{i}\right)$ constructed by folding flux data are averages of the actual pulse profiles over the time interval required to construct a stable profile, which can be hundreds or even thousands of seconds, $10^{5}-10^{6}$ times longer than the $\sim 1 \mathrm{~ms}$ dynamical timescale near the neutron star. The folded pulse profiles are therefore likely to vary more slowly and have less detail than the X-ray waveform, a point to which we will return in Section 4.

The waveforms of AMXPs can be modeled even on the dynamical timescale near the stellar surface, but such waveforms would contain much more information than can be studied using current observations. Consequently, we focus here on modeling folded pulse profiles. We define a computed pulse profile as the waveform seen by a given observer when a star with a constant

\footnotetext{
6 The waveforms reported by Pechenick et al. (1983) for two antipodal spots are slightly inaccurate, as shown by the following two tests. The waveform seen by an observer in the star's rotation equator viewing two identical antipodal spots in the rotation equator should be the same at $180^{\circ}$ as at $0^{\circ}$, but this is not the case for their waveform for this geometry (see their Figure 7). More generally, the flux from a uniform, isotropically emitting, circular spot on a spherical, nonrotating star should depend only on the angle between the radius through the center of the spot and the radius to the observer. This is not quite true for the waveforms reported by Pechenick et al. (1983). The waveforms given by our code pass these tests (for details, see S. Boutloukos et al. 2009, in preparation).
} 
emission pattern makes one rotation. It is customary and useful to describe pulse profiles by the amplitudes and phases of their Fourier components. We describe our computed profiles using the representation

$$
W_{P}(t)=A_{0}+\sum_{k=1}^{\infty} A_{k} \cos \left[2 \pi k\left(v_{s} t-\phi_{k}\right)\right]
$$

Here $t$ is the time measured at infinity, $A_{0}$ is the average of the flux over one rotation period, $A_{k}$ and $\phi_{k}$ are the amplitude and phase of the $k$ th harmonic component of the pulse, and $v_{s}$ is the stellar rotation frequency. For harmonic $k$, the range of unique phases is 0 to $1 / k$. We sometimes also refer to the phase $\phi_{p}$ of the peak of the pulse profile, defined in a similar way. With these definitions, shifting the arrival time of a given pulse by an amount $\delta t$ shifts the phases of its Fourier components and the phase of the pulse peak by $v_{s} \delta t$ cycles.

We define the arrival time $t_{k}$ of the $k$ th harmonic component of the pulse profile to be zero if the first maximum of the sinusoid associated with this component reaches the observer at the same time as would a light pulse coming from a point on the stellar surface immediately below the observer (see Section 2.2). The arrival time $t_{k}$ is affected by the sizes, shapes, and locations of the emitting areas, the beaming pattern of the emitted radiation, and the aberration and Doppler shifts produced by the spin of the star and usually is not zero. We define the arrival phase of the $k$ th harmonic component by $\phi_{k}=v_{s} t_{k}$. The arrival time $t_{p}$ and arrival phase $\phi_{p}$ of the peak of the pulse profile are defined similarly. We refer to $t_{k}$ and $t_{p}$ as time residuals and to $\phi_{k}$ and $\phi_{p}$ as phase residuals. An increase in a time or phase residual indicates that the harmonic component or the peak is arriving later.

\section{OSCILLATION AMPLITUDES}

As discussed in Section 1, the fractional amplitudes of the accretion-powered oscillations of most AMXPs are typically $\sim 1 \%-2 \%$, but the amplitudes of several AMXPs are sometimes as large as $\sim 10 \%-20 \%$. A successful model of the accretionpowered oscillations of the AMXPs should therefore be able to explain oscillation amplitudes as low as $\sim 1 \%-2 \%$ without requiring a special viewing angle or stellar structure and should also be able to explain the higher amplitudes sometimes seen.

As was also discussed in Section 1, the accretion-powered oscillations of the AMXPs are often nearly sinusoidal. The second harmonic of the fundamental oscillation frequency has been detected in seven of the 10 known AMXPs, but it is typically $\gtrsim 10$ times weaker than the fundamental, although in a few cases it is not this weak and in one case, SAX J1808.4-3658, it is sometimes stronger than the fundamental. Upper limits $\sim 0.1 \%-0.2 \%$ have been placed on the amplitude of any second harmonic in three AMXPs.

Upper limits $\sim 0.5 \%-1 \%$ have been placed on the fractional amplitudes of accretion-powered millisecond oscillations in several AMXPs that produce nuclear-powered millisecond oscillations, for frequencies close to the frequencies of the nuclear-powered oscillations. A model that can explain these nondetections as well as the observed properties of the detected accretion-powered oscillations would be very attractive.

In this section, we show that emitting regions on or near the stellar surface can produce oscillation amplitudes as low as $\sim 1 \%-2 \%$ for a substantial range of viewing directions only if they are located within a few degrees of the stellar spin axis. Regions near the spin axis also naturally produce nearly sinusoidal pulse profiles. If they are very close to the spin axis and remain there, the amplitudes of the oscillations they produce can be $\sim 0.5 \%$ or less. Thus, emission from very close to the spin axis, in combination with other effects, such as rapid phase and amplitude fluctuations and suppression of X-ray modulation by scattering of photons in circumstellar gas (see Lamb et al. 1985; Miller 2000), may explain the nondetection of accretionpowered oscillations in accreting neutron stars in which nuclearpowered oscillations have been detected.

We show further that although the fractional oscillation amplitudes produced by large regions tend to be smaller, this is a weak effect. Unless almost the entire surface of the star is uniformly emitting, amplitudes as small as those observed are possible even for large spots only if they are located near the stellar spin axis. We also show that although the fractional amplitudes produced by very compact neutron stars tend to be smaller than the amplitudes produced by less compact stars, this effect is too weak to explain the small amplitudes of the AMXPs. Furthermore, it cannot explain why the fractional amplitudes of several AMXPs are $\sim 1 \%-2 \%$ at some times but $\sim 15 \%-$ $25 \%$ a few hours or days later, since the stellar compactness cannot change on such short timescales. Finally, we note that the fractional amplitudes produced by spinning neutron stars depend only weakly on their spin rates.

Measured oscillation amplitudes are likely to be smaller than those shown in the figures in this section, which are for stable spots fixed on the stellar surface. The reason is that the pulse shape is expected to vary on timescales shorter than the time needed to construct a pulse waveform. Such rapid pulse shape variations will appear as increased background noise, reducing the apparent amplitude of the oscillations (see Lamb et al. 1985). As discussed below, this effect tends to be more important when the emitting area is near the spin axis, and it will therefore tend to reduce further the apparent amplitude of the oscillations produced by spots at small inclinations.

In Section 4, we show that the variations of the oscillation amplitudes seen in all AMXPs and the larger fractional amplitudes $\sim 15 \%-20 \%$ occasionally seen in several of them can be explained by modest increases in the inclinations of their emitting regions, if these regions are close to the star's spin axis.

\subsection{Dependence on Spot Inclination}

The precise distance emitting regions can be from the spin axis and still produce oscillation amplitudes as low as the $\sim 1 \%-2 \%$ amplitudes often observed in the AMXPs depends on the beaming pattern of the emission. The angular pattern of the radiation flux produced by Thomson scattering is strongly peaked in the direction normal to the stellar surface, whereas the flux patterns produced by fan-shaped radiation beams like those proposed by Poutanen \& Gierliński (2003) to describe Comptonized emission from near the stellar surface are peaked much less (if at all) in the direction normal to the stellar surface. The angular pattern of the radiation flux produced by isotropic emission is intermediate between these two cases, being peaked normal to the surface less than the pattern produced by Thomson scattering but more than the pattern produced by fan-beam emission. We first discuss the waveforms produced by isotropic emission and then the waveforms produced by other beaming patterns.

Figure 1 shows the fractional rms amplitudes of the first harmonic (fundamental) and second harmonic (first overtone) components of the pulse profile and the total fractional rms amplitude produced by isotropic emission from a single stable 

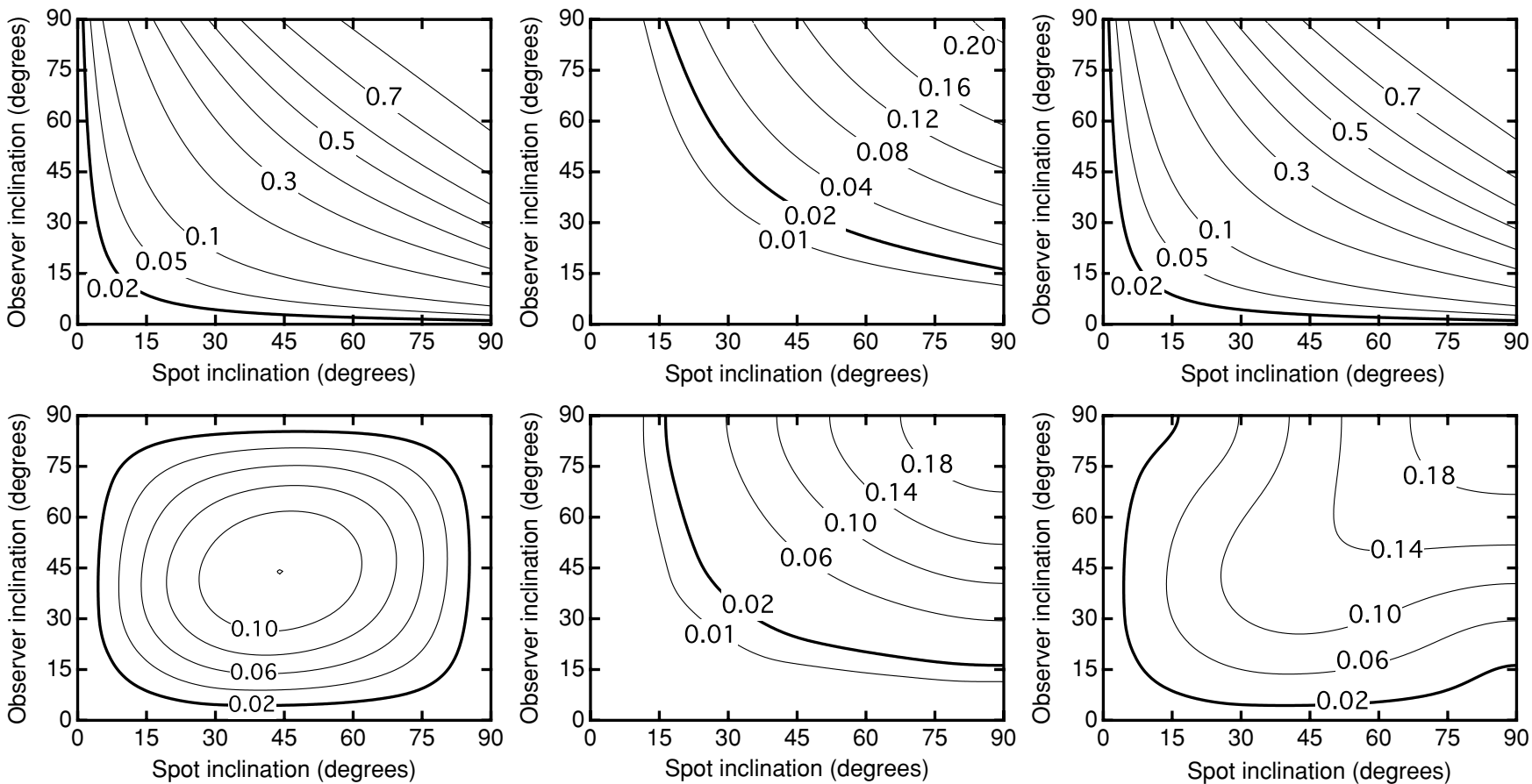

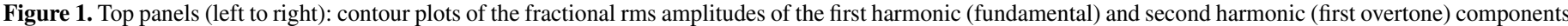

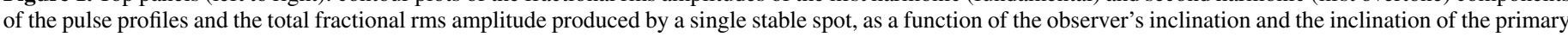

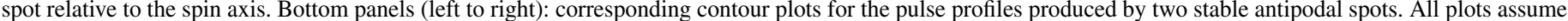

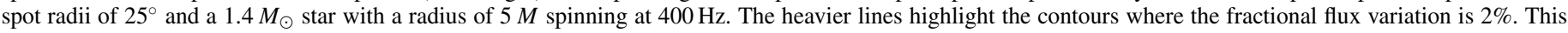

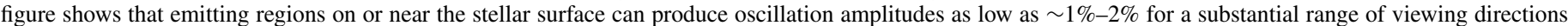
only if they are located within a few degrees of the stellar spin axis.

spot and from two stable antipodal spots, as a function of the observer's inclination and the inclination of the primary spot relative to the spin axis. The curves are for spots with angular radii of $25^{\circ}$ on our reference star (a $1.4 M_{\odot}$ star with a radius of $5 \mathrm{M}$ spinning at $400 \mathrm{~Hz}$ ). As expected, all amplitudes are zero when the star is viewed along its spin axis $\left(i=0^{\circ}\right)$ or the emitting spots are centered on the rotation pole $\left(i_{s}=0^{\circ}\right.$ or $i_{s 1}=0^{\circ}$ and $\left.i_{s 2}=180^{\circ}\right)$. In addition, the amplitude of the first harmonic variation produced by two stable antipodal spots vanishes if either the observer or the spots are in the rotation equator $\left(i_{s}=90^{\circ}\right.$ or $\left.i_{s 1}=i_{s 2}=90^{\circ}\right)$, whereas the amplitude of the second harmonic is highest for these geometries.

The top panels in Figure 1 show that if the observer sees radiation only from a single spot, the total fractional amplitude can be as low as $\sim 1 \%-2 \%$ for a range of viewing angles only if the spot is within a few degrees of the spin axis. These panels also show that for radiation from a single spot, the second harmonic is $\sim 5-10$ times smaller that the first harmonic for most observer and spot inclinations.

The bottom panels in Figure 1 show that if the observer sees radiation from two antipodal spots, the total fractional amplitude can be as low as $\sim 2 \%$ for a substantial range of viewing angles only if the spots are within about $5^{\circ}$ of the spin axis. These panels also show that for radiation from two antipodal spots, the second harmonic is smaller than the first harmonic for observer or spot inclinations less than about $60^{\circ}$. The amplitude of the second harmonic is $\lesssim 2 \%$ for all observers only if the spots are within $15^{\circ}$ of the spin axis.

Two emitting spots centered near the same rotation pole are expected if the inward motion of neutron vortices in the stellar core drives both of the star's magnetic poles toward the same rotation pole (see Section 5.1). The fractional modulation produced by two spots with this geometry is typically somewhat larger than the modulation produced by two antipodal spots if the observer and spot inclinations are both large, but is typically smaller than the modulation produced by two antipodal spots if the observer inclination or the spot inclination is small. Even so, the total fractional amplitude can be as low as $\sim 1 \%-2 \%$ for a range of viewing angles only if the spots are within a few degrees of the stellar spin axis. For example, for our reference star the fractional modulation produced by two spots at the same inclination but separated by $160^{\circ}$ in longitude is larger than the modulation produced by two antipodal spots if $i \gtrsim 70^{\circ}$ and $i_{s 1}=i_{s 2} \gtrsim 70^{\circ}$ but is smaller if $i \lesssim 60^{\circ}$ and $i_{s 1}=i_{s 2} \lesssim 60^{\circ}$. The total fractional amplitude can appear as low as $\sim 1 \%-2 \%$ over half the sky $\left(i \geqslant 60^{\circ}\right)$ only if the two spots are within $\sim 10^{\circ}$ of the spin axis.

The intensity distribution produced by a Thomson scattering atmosphere is described by a linear combination of Hopf functions (see Chadrasekhar 1960, Section 68) and peaks in the direction normal to the stellar surface. The radiation flux from such an atmosphere is given by the product of this intensity distribution and the projected area of the surface, which is proportional to the cosine of the angle to the normal. Consequently, the radiation flux from a Thomson scattering surface is strongly peaked in the direction normal to the surface. For this reason, emission from a Thomson scattering region typically produces a higher modulation fraction than would isotropic emission from the same region. Hence if Thomson scattering is important, the emitting regions must be closer to the star's spin axis than if the emission is isotropic.

Fan-shaped intensity distributions like those proposed by Poutanen \& Gierliński (2003) to describe Comptonized emission from near the stellar surface can partially compensate for the decrease of the projected area as the angle from the normal to 

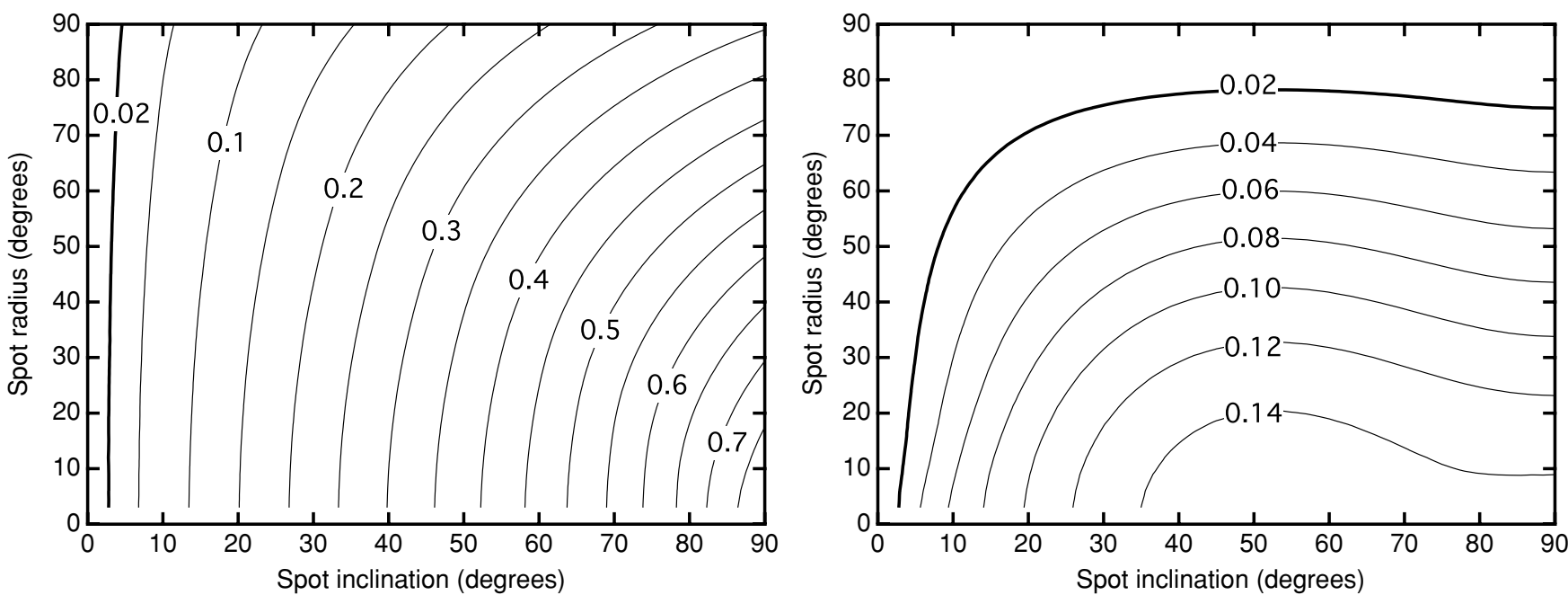

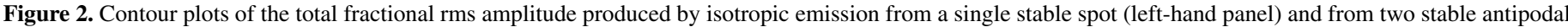

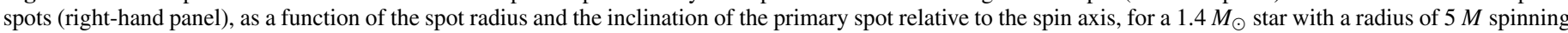

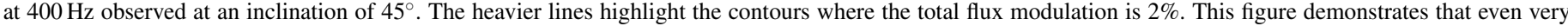

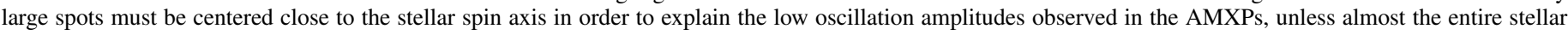
surface is uniformly emitting.

the surface increases, producing a radiation flux distribution that is more isotropic than that produced by an isotropic intensity distribution. For this reason, fan-shaped emission from a single spot can produce modulation at the first harmonic of the spin frequency that is smaller than would be produced by isotropic emission from the same spot. A spot producing this emission pattern must still be close to the spin axis in order for the amplitude of the first harmonic to be $\lesssim 2 \%$. Fan-shaped emission from a single spot tends to increase the amplitude of the second harmonic relative to the first. Fan-like emission from two antipodal spots produces waveforms similar to those produced by isotropic emission, but the modulation fraction can be up to a factor of 2 larger.

In SAX J1808.4-3658, the second harmonic is usually weak but is occasionally as strong as or even stronger than the first harmonic (see Hartman et al. 2008). The strengthening of the second harmonic could be caused by development of a twofold asymmetry of one spot, by more fan-beam emission from a single spot, or by increased visibility a second, antipodal spot.

Our computations show that modulation fractions are below typical current detection limits if the emitting regions are within $1^{\circ}$ of the spin axis and remain there. As discussed in Section 4, rapid fluctuations in the amplitude and phase of the oscillations would not be directly detectable in time-averaged observations, but would raise the background noise level and further reduce the detectability of the oscillations. These effects, possibly in combination with others, such as reduction of the modulation fraction by scattering in circumstellar gas (Lamb et al. 1985; Miller 2000), may explain the nondetection of accretion-powered oscillations in accreting neutron stars in which nuclear-powered oscillations have been detected.

\subsection{Dependence on Spot Size}

Larger emitting spots tend to produce lower oscillation amplitudes than smaller spots, but even very large spots must be centered close to the stellar spin axis in order to explain the low oscillation amplitudes observed in the AMXPs, unless almost the entire stellar surface is uniformly emitting. This is illustrated in Figure 2, which shows the total fractional amplitude produced by isotropic emission from a single spot and from two antipodal spots, as a function of the radius of the spots and the inclination of the primary spot relative to the spin axis. All the curves in this figure are for our reference star, viewed at an inclination of $45^{\circ}$.

The left-hand panel of Figure 2 shows that even a single spot that covers half of the star (a spot with a radius of $90^{\circ}$ ) can produce a total fractional modulation as small as $2 \%$ only if its inclination is $\lesssim 5^{\circ}$. The modulation fraction depends only weakly on the size of the spot, especially for small-to-moderate spot inclinations. For example, the amplitude of the oscillation produced by a single spot inclined $45^{\circ}$ from the spin axis decreases by only $\sim 10 \%$ as the spot radius increases from $5^{\circ}$ to $45^{\circ}$. Even for a spot inclined $90^{\circ}$ from the spin axis, the fractional flux modulation depends only weakly on the size of the spot for spot radii $\lesssim 50^{\circ}$, decreasing from $70 \%$ to $35 \%$ as the spot radius increases from $30^{\circ}$ to $90^{\circ}$. The reason for this insensitivity is that the angular width of the stellar radiation pattern produced even by spots with radii $\sim 50^{\circ}$ is governed mostly by the beaming pattern of the radiation from the stellar surface and the magnitude of the gravitational light deflection, not by the radius of the spot. A single spot at an inclination of $70^{\circ}$ can produce a fractional modulation $\lesssim 2 \%$ only if the entire surface of the star is uniformly emitting except for a dark area of radius $20^{\circ}$ on the opposite side of the star from the center of the spot (i.e., if the spot radius is $\gtrsim 160^{\circ}$ ).

The right-hand panel of Figure 2 shows that two uniformly emitting antipodal spots of a given radius produce a smaller fractional modulation than a single spot of the same radius, because the antipodal spots cover twice as much of the stellar surface. Even so, the antipodal spots must be very large in order to produce fractional modulations as small as those observed. For example, two antipodal spots on our reference star can produce a fractional modulation as low as $\sim 2 \%$ for most observing directions only if they have radii $\gtrsim 75^{\circ}$, which means that all of the stellar surface is uniformly emitting except for a band around the star with a total width $\lesssim 30^{\circ}$. The fractional modulation for most observing directions doubles to $\sim 4 \%$ if the spot radius decreases from $75^{\circ}$ to $65^{\circ}$.

These results show that the low fractional amplitudes often observed in the AMXPs cannot be explained by large emitting 

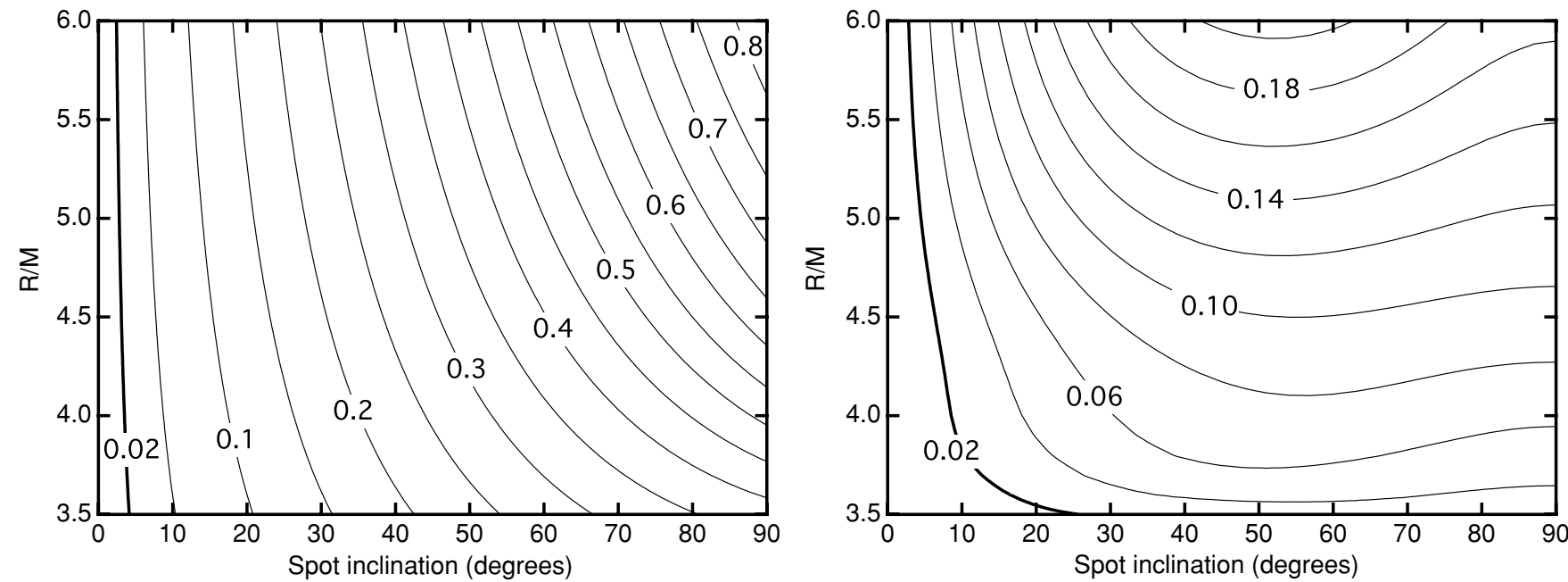

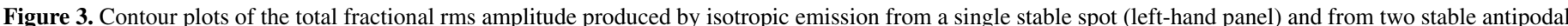

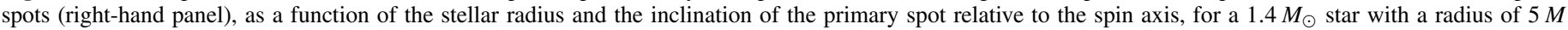

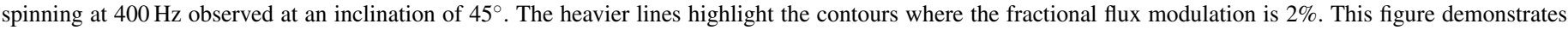
that compactness alone cannot explain the low-amplitude oscillations of the AMXPs.

regions, unless the emitting regions are uniform and cover almost the entire stellar surface, which is not expected. Another difficulty with attributing the low fractional amplitudes often observed to large emitting areas is that a substantial number of AMXPs that are observed to have fractional amplitudes $\sim 1 \%-$ $2 \%$ at some times are observed to have much larger fractional amplitudes $\sim 15 \%-20 \%$ at other times. Hence attributing their small amplitudes at some times to large emitting areas would require their emitting areas to shrink by a large factor at other times, which is not expected.

\subsection{Dependence on Stellar Compactness}

The fractional modulation produced by a given emission pattern is generally smaller for more a compact star (see, e.g. Pechenick et al. 1983; Strohmayer 1992). The reason is that gravitational focusing allows the observer to see a larger fraction of the surface of such a star, averaging the contributions of its bright and dark areas and reducing the flux variation seen as the star turns. Strong gravitational focusing can increase the fractional modulation seen by some observers if the star is very compact $(R \lesssim 3.5 M)$.

Even if all AMXPs are relatively compact $(R \sim 4 M)$, which is not expected, they would produce fractional modulations larger than the $\sim 1 \%-2 \%$ fractional modulations often observed (see Section 1) unless their emitting regions are close to their stellar spin axes. Nor can the failure so far to detect accretionpowered oscillations in some nuclear-powered AMXPs be explained unless the accretion-powered emission comes from areas very close to the stellar spin axis. These points are illustrated by Figure 3, which shows the fractional amplitudes produced by isotropic emission from a single spot (left-hand panel) and from two antipodal spots (right-hand panel) as a function of $R / M$ and the inclination of the primary spot relative to the spin axis, for a $1.4 M_{\odot}$ star spinning at $400 \mathrm{~Hz}$ observed at an inclination of $45^{\circ}$.

The left-hand panel of Figure 3 shows that even if all the AMXPs had radii as small as $4 M$, their fractional modulations could be as small as $\sim 2 \%$ for emission from a single spot only if the spot is within $3^{\circ}$ of the spin axis. If instead the spot is at a moderate-to-high inclination, their fractional modulations would be much larger. For example, if the spot is $45^{\circ}$ from the spin axis, the fractional modulation would be $\sim 25 \%$.

The right-hand panel of Figure 3 shows that although the fractional modulation produced by two antipodal spots is generally smaller than the modulation produced by a single spot, antipodal spots would have to be within $10^{\circ}$ of the spin axis to produce a fractional modulation as small as $\sim 2 \%$, even for a star as compact as $4 \mathrm{M}$. If instead the spots are $45^{\circ}$, from the spin axis, the fractional modulation would be $\sim 7 \%$, much larger than the lowest modulations observed in most AMXPs.

As another example (not plotted here), consider a relatively massive $2.2 M_{\odot}$ star with one or two spots that are not close to the spin axis, observed at an inclination of $45^{\circ}$. If there is a single, isotropically emitting spot of radius of $25^{\circ}$ at an inclination of $45^{\circ}$, the fractional modulation would be $\sim 30 \%$ if the star has a radius of $4 M$ and $22 \%$ if the star has a radius of $3.2 \mathrm{M}$. If instead there are two antipodal, isotropically emitting spots at inclinations of $45^{\circ}$ and $135^{\circ}$, the fractional modulation would be $\sim 11 \%$ if the star has a radius of $4 M$ and $9 \%$ if the star has a radius of 3.2 $\mathrm{M}$. These fractional modulations are much larger than the lowest modulations observed in most AMXPs. The fractional modulation is typically even larger for stars that are still more compact, because of the strong gravitational focusing effect discussed above.

These results show that even if the AMXPs are compact neutron stars, their fractional modulation will be much larger than the low fractional modulations they often produce, unless their emitting areas are within a few degrees of the spin axis. Hence the low fractional modulations often observed in the AMXPs cannot be attributed to high stellar compactness.

A further difficulty in attributing the generally low fractional modulations of the AMXPs to high stellar compactness is that several of the AMXPs that exhibit fractional modulations $\sim 1 \%-$ $2 \%$ at some times exhibit fractional modulations $\sim 15 \%-25 \%$ only a few hours or days later. The compactness of the neutron star cannot change significantly on such short timescales, and hence some mechanism other than high compactness must be responsible for the low fractional modulations seen at many times in most AMXPs.

Our results also show that high stellar compactness cannot be the main factor responsible for the nondetection of accretion- 

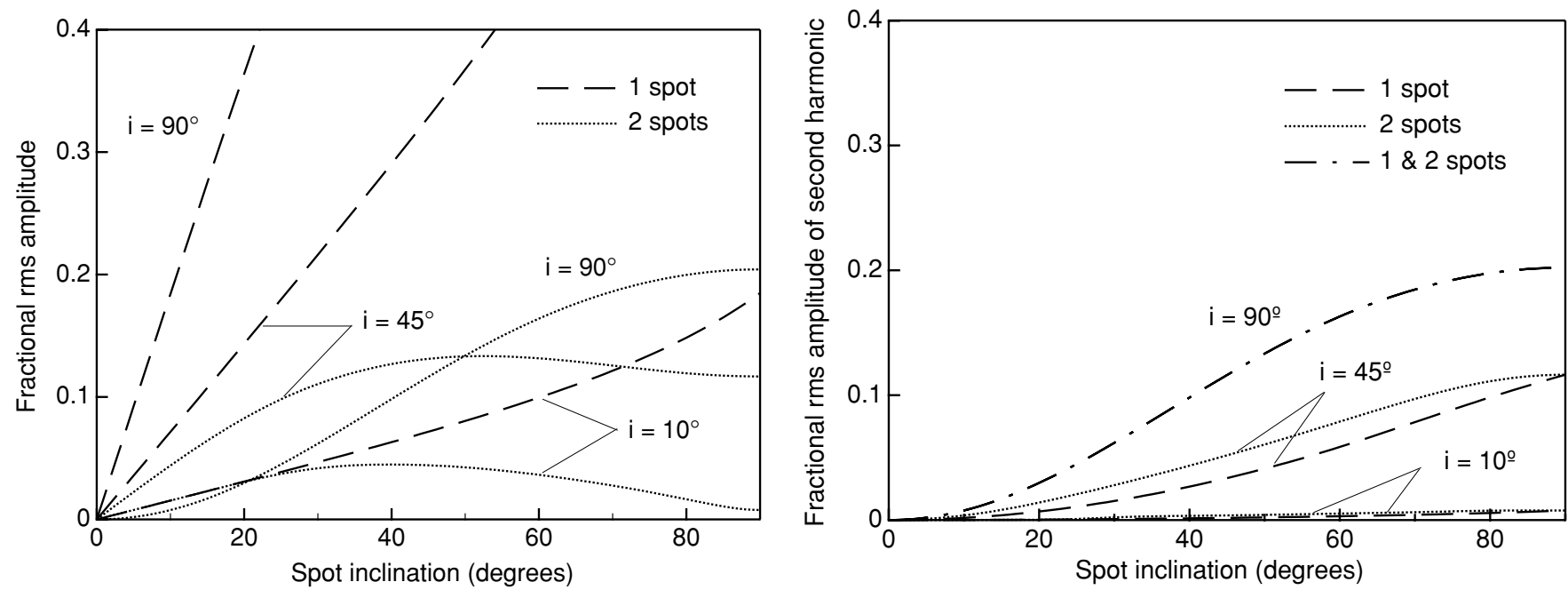

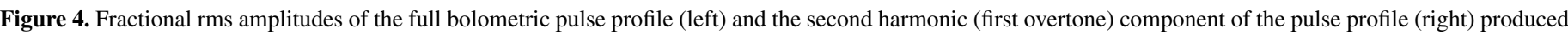

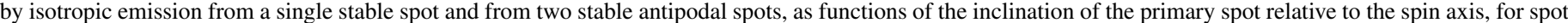

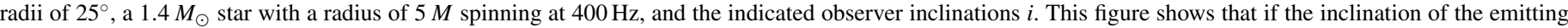
region is small, modest changes in its inclination can produce large changes in the observed amplitude of the oscillation.

powered oscillations in many accreting neutron stars in lowmass X-ray binary systems, including many stars in which nuclear-powered oscillations have been detected. The upper limits on the fractional amplitude of any accretion-powered oscillation produced by these stars are $\lesssim 0.5 \%$ (see Section 1 ), much less than could be explained by the effects of high stellar compactness.

\subsection{Dependence on Stellar Spin Rate}

Most of the results discussed in this section are for stars spinning at $400 \mathrm{~Hz}$. Other things being equal, stars spinning more rapidly will produce oscillations with larger fractional amplitudes, because their higher surface velocities will produce larger Doppler boosts and greater aberration, making their radiation patterns more asymmetric (Miller \& Lamb 1998; Braje et al. 2000). Conversely, stars spinning more slowly tend to produce oscillations with smaller fractional amplitudes. However, the dependence of the fractional amplitude on the stellar spin rate is weak. Consequently, the basic conclusions reached in this section are valid for the full range of AMXP spin rates observed.

\section{PULSE AMPLITUDE AND PHASE VARIATIONS}

As discussed in Section 1, the fractional amplitudes of the accretion-powered oscillations of the AMXPs vary by factors ranging from $\sim 2$ to $\sim 10$ on timescales of hours to days. As was also discussed in Section 1, the phases of the Fourier components of these oscillations vary by $\sim 0.1-0.4$ cycles on similar timescales. If interpreted as caused by changes in the stellar spin rate, the observed phase variations would imply frequency variations more than a factor of 10 larger than expected for the largest accretion torques and the smallest inertial moments thought possible for these stars. In several AMXPs, the phase variations are correlated with the amplitude variations, for some amplitude ranges. A successful model of the accretion-powered oscillations of the AMXPs should provide a consistent explanation of these properties of the oscillations.

As noted in Section 1, changes in the latitude and longitude of the emitting area are expected on a wide range of timescales, from the $\sim 0.1 \mathrm{~ms}$ dynamical time at the stellar surface to the $\sim 10$ day timescales of the variations observed in the mass accretion rates of the AMXPs. Changes in the latitude (inclination) of the emitting area primarily affect the amplitudes of the harmonic components of the pulse profile but also affect their phases. Modest changes in the latitude of the emitting area can produce large changes in the oscillation amplitude if the emitting region is close to the stellar spin axis. Changes in the longitude (stellar azimuth) of the emitting area shift the phases of the harmonic components of the pulse but do not affect their amplitudes.

In this section, we show that if the emitting area is close to the spin axis, the fractional amplitude variations seen in all AMXPs and the larger fractional amplitudes $\sim 15 \%-20 \%$ occasionally seen in several of them can be explained by modest variations of the inclinations of their emitting areas. We show further that if the emitting area is close to the spin axis and moves in the azimuthal direction by even a small distance, the phases of the Fourier components of the pulse will shift by a large amount. These shifts can easily be as large as $\sim 0.1-0.4$ cycles. Indeed, if the emitting area loops the spin axis, the phases of the Fourier components will shift by more than one cycle. We show that changes in the latitude and longitude of the emitting area by modest amounts can produce substantial correlated changes in the amplitudes and phases of the Fourier components of the oscillation.

\subsection{Pulse Amplitude Variations}

Figure 4 shows the total fractional rms amplitude and the fractional rms amplitude of the second harmonic (first overtone) of the spin frequency for pulses produced by isotropic emission from a single stable spot and from two stable antipodal spots, as functions of the inclination of the primary spot relative to the spin axis. These curves are for spots with angular radii of $25^{\circ}$ on our reference star.

The results shown in Figure 4 support our conclusion in Section 3 that the oscillation amplitudes seen by most observers can be as small as $\sim 1 \%-2 \%$ only if the emitting regions are within a few degrees of the spin axis. If instead the emitting regions were $\gtrsim 45^{\circ}$ from the spin axis, most observers would see modulation fractions $\gtrsim 15 \%$. 
Whether the observer sees emission from a single area or from two antipodal areas, the total fractional amplitude of the oscillation will increase if the inclination of an emitting region initially at a low inclination increases. The reason is that the star's radiation pattern becomes steadily more asymmetric about the spin axis as the emitting region moves farther from the spin axis, partly because the emitting region becomes more asymmetric and partly because the surface velocity becomes higher.

If the emitting area is initially close to the spin axis, a modest increase or decrease in its latitude can change the amplitude of the observed flux oscillation by a substantial factor. For emission from a single spot, the amplitude increase is steepest for observers at high inclinations, whereas for emission from two antipodal spots, the amplitude increase is steepest for observers at moderate inclinations.

These effects are illustrated by the left-hand panel of Figure 4 . It shows that for a single spot initially at $i_{s}=10^{\circ}$, an increase of the spot inclination by as little as $\sim 5^{\circ}$ can increase the fractional amplitude of the oscillations seen by an observer at $45^{\circ}$ by a factor of 2. For observers at higher inclinations, the oscillation amplitude increases even more.

A single spot at a high inclination produces oscillations with a high fractional amplitude for observers at inclinations $\gtrsim 60^{\circ}$ and the amplitude increase only slightly as the inclination increases further. As an example, for the parameter values assumed in Figure 4, the total fractional amplitude seen by an observer at $i=45^{\circ}$ increases steadily with increasing spot inclination, reaching $\sim 70 \%$ when $i_{s}=90^{\circ}$. In contrast, the fractional amplitude seen by an observer at $i \approx 90^{\circ}$ (i.e., close to the spin equator) is $\sim 85 \%$ for $i_{s}=70^{\circ}$ and $\sim 90 \%$ for $i_{s}=90^{\circ}$.

If instead the observer sees radiation from two antipodal spots, the total fractional amplitude increases with spot inclination for spot inclinations $\lesssim 50^{\circ}$, though not as steeply as for a single spot. The fractional amplitude eventually decreases with increasing spot inclination, unless the observer is located in the spin equator. A change in the inclination of two antipodal spots by $10^{\circ}$ can change the total fractional amplitude seen by an observer at $45^{\circ}$ by a factor of 2 .

The right-hand panel of Figure 4 shows that the fractional amplitude of the second harmonic is almost the same for radiation from one spot or from two antipodal spots, independent of the inclinations of the observer and the spot(s). Comparison of the right-hand panel of Figure 4 with the left-hand panel shows that the second harmonic dominates the pulse profile for spot inclinations $\lesssim 80^{\circ}$.

The accretion-powered oscillations observed in XTE J1814-338 and XTE J1807-294 occasionally have fractional amplitudes as large as 11\% and 19\%, respectively, although they are usually much smaller (Chung et al. 2008; Patruno 2008; Zhang et al. 2006; Chou et al. 2008). Figure 4 shows that amplitudes this large are possible for isotropic emission from a single spot even for relatively small spot inclinations, provided the observer's line of sight is not too close to the star's spin axis. For example, amplitudes this large are possible for spot inclinations $\sim 5^{\circ}-15^{\circ}$ if the observer's inclination is $250^{\circ}$. A decrease in the spot inclination to $\sim 1^{\circ}-3^{\circ}$ would then reduce the fractional amplitude to $\sim 1 \%-3 \%$, the lowest values observed in these AMXPs.

If the emitting areas of the AMXPs are typically located at high stellar latitudes, as in the model discussed here, their pulse amplitudes are expected to depend on the accretion rate and structure of the inner disk. These may vary on timescales as short or shorter than the $\sim 0.1 \mathrm{~ms}$ dynamical time at the stellar surface up to timescales as long as the $\sim 10$ day observed variations of the mass accretion rate. Consequently, the harmonic amplitudes of AMXP pulses are expected to vary on these timescales.

This model can be tested on timescales longer than the $\sim 10^{2}$ $10^{3} \mathrm{~s}$ integration times required to construct pulse profiles by searching for correlations between the amplitudes of the harmonic components of the pulse profile or the variance of the pulse amplitude and the pulsar's X-ray flux or spectrum. Indeed, the rms fractional amplitudes of the pulses of XTE J1814-338 appear to be positively correlated with its mean Xray flux (Chung et al. 2008). Methods for testing the model on timescales shorter than the time required to construct a pulse profile are discussed in Section 4.5.

\subsection{Pulse Phase Variations}

A change in the longitude (stellar azimuth) of the emitting region alters the arrival time of the pulse. This shifts the measured phases of all the Fourier components by the same amount, relative to their phases if the pulse, were produced by an emitting area fixed on the surface of a star rotating at a constant rate. Obviously, the phase of the pulse peak is also shifted by this amount. If the emitting area is close to the spin axis, a displacement in the azimuthal direction by even a small distance can produce large phase shifts.

A change in the latitude (inclination) of the emitting area not only alters the amplitudes of the Fourier components of the pulse but also their arrival times (phases). These alterations change the shape and arrival time of the pulse in a complex way. They occur because a change in the latitude of the emitting area changes the velocity of the emitting surface, altering the aberration, Doppler shift, and propagation time of each photon. The resulting changes in the pulse shape and arrival time depend on the beaming pattern of the radiation, the properties of the star, and the inclination of the observer. The phase shifts caused by changes in the latitude of the emitting area are expected to be modest, because the surface velocities of neutron star models with spin frequencies $\lesssim 600 \mathrm{~Hz}$ are typically a small fraction of the speed of light.

Figure 5 shows how the phases of the peak of the pulse and its first and second harmonic components vary as the emitting spot moves along a circular path on the stellar surface. Motion along this path changes both the latitude and the longitude of the spot. Motion in either direction along all or part of such a path may occur if the main impact area of the accreting matter moves around the magnetic pole as a result of changes in the structure of the inner disk, changes that could be caused by variations of the accretion rate onto the star (see Section 2.1).

The left-hand panel of Figure 5 introduces the angles used to describe the motion of the spot around the path. The righthand panel shows the changes in the arrival times and phases of the peak of the pulse and its Fourier components that occur as the spot moves around the path shown in the left-hand panel. In this example, the phases of the peak and the first and second harmonic components vary by $\sim 0.35$ cycles, which is comparable to the phase variations seen in XTE J1807-294 (Markwardt 2004) and SAX J1808.4-3658 (Morgan et al. 2003; Hartman et al. 2008) on timescales of hours to days. If the path of the emitting region approaches or loops the spin axis, even a very small displacement can shift the phases by more than one cycle. The phase shifts shown in Figure 5 are typical for isotropic emission. The phase shifts for Comptonized-emission- 

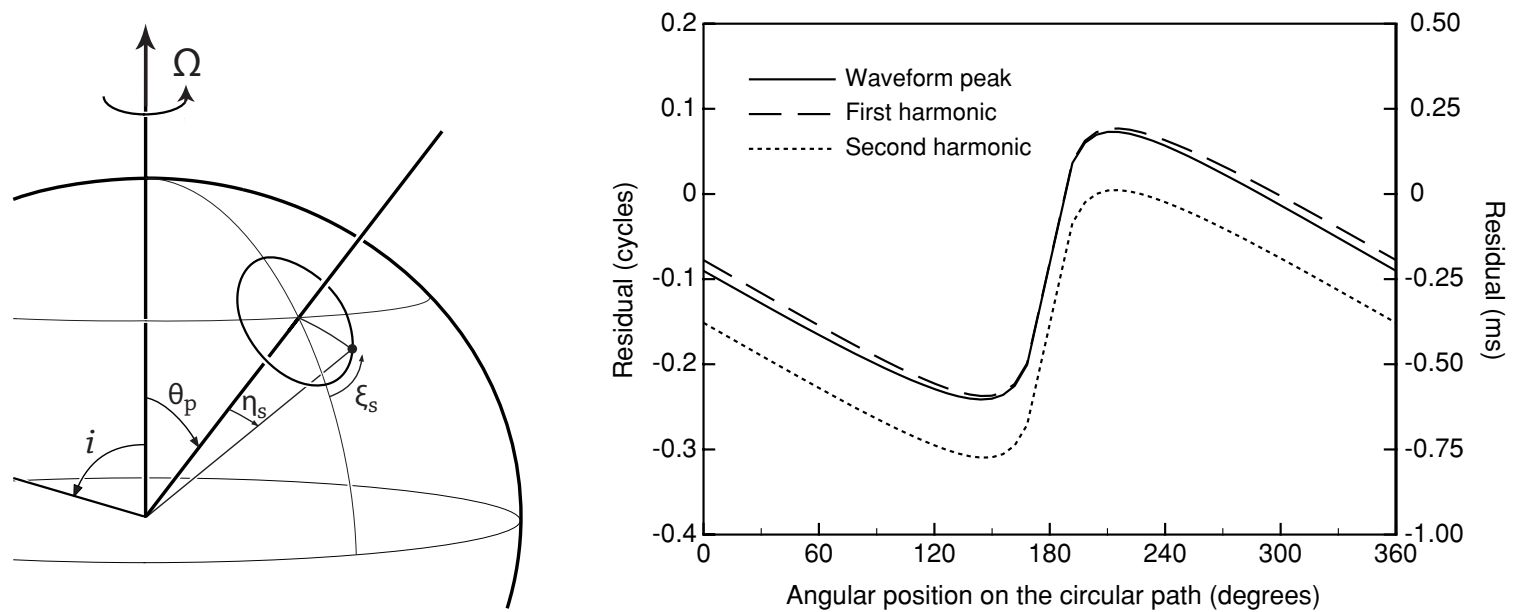

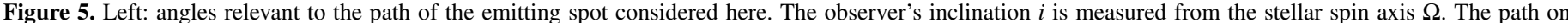

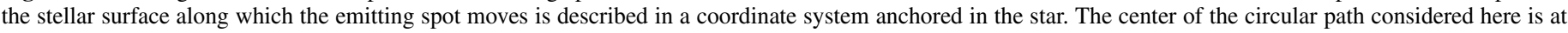

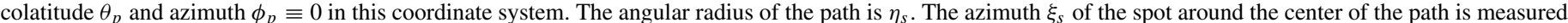

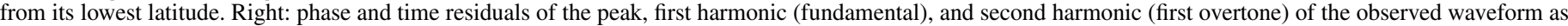

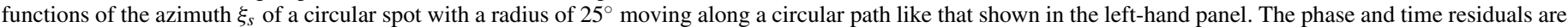

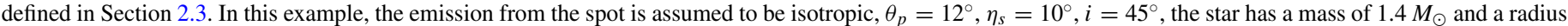
of $5 \mathrm{M}$, and the star's spin rate is $400 \mathrm{~Hz}$ measured at infinity.

beaming patterns like those proposed by Poutanen \& Gierliński (2003) are similar, but differ in detail.

The phase and time residuals shown in Figure 5 are not zero when the spot is in the plane defined by the spin axis and the observer $\left(\xi_{s}=0\right)$ for two reasons. First, when $\xi_{s}=0$ the spot is not directly beneath the observer in this example, so a photon emitted at this moment will reach the observer later than would a photon emitted from the point directly beneath the observer, which is the emission point that defines the zero of the arrival time at the observer (see Section 2.3). Second, the spinning motion of the stellar surface produces aberrations, time delays, and Doppler energy shifts that distort the pulse, causing its harmonic components to reach the observer at times different from their arrival times from a nonrotating star. In this particular example, these effects cause the peak of the pulse and its harmonic components to arrive earlier than they would if the star were not rotating. The phase advance produced by these effects more than offsets the backward phase shift produced by the extra travel time.

As the emitting spot moves around the path shown in Figure 5 , its inclination increases from $2^{\circ}$ to $22^{\circ}$. This causes the amplitude of the oscillation to increase from $\sim 1 \%$ when the spot is closest to the rotation pole to $\sim 15 \%$ when it is farthest from the pole. This variation is comparable to the amplitude variations observed in XTE J1814-338 and XTE J1807-294 (Chung et al. 2008; Patruno 2008; Zhang et al. 2006; Chou et al. 2008).

As described above, the change in the inclination of the spot as it moves around the path shown in Figure 5 also contributes to the phase shifts. In general, an increase in the inclination of an emitting spot by $20^{\circ}$ can by itself shift the phases of the Fourier components of the pulse by amounts ranging from $\sim 0.05$ to $\sim 0.20$ cycles, depending on the beaming pattern, stellar properties, and viewing direction, and can shift the phases of different harmonic components by different amounts. These effects are included in the phase shifts shown in Figure 5.

These results show that if the emitting areas of the AMXPs are usually near their spin axes, as in the model discussed here, movement of the emitting area by modest distances can cause the arrival times of pulse Fourier components to vary by the large amounts observed, even if the AMXPs spin at a nearly constant rate.

Emitting areas occur where accreting plasma impacts the stellar surface. As discussed in Section 2.1, the position of these areas depends on how accreting plasma enters the magnetosphere and hence on the accretion rate and the structure of the inner disk. Consequently, pulse phase variations produced by changes in the location of the emitting areas should be correlated with changes in the luminosity and spectrum of the pulsar. The properties of the accretion flow are expected to vary on timescales ranging from as short as $\sim 0.1 \mathrm{~ms}$ to as long as $\sim 10$ days, and the arrival times of pulse Fourier components should therefore vary on these timescales also.

This model of pulse phase variations can be tested on timescales longer than the $\sim 10^{2}-10^{3}$ s times required to construct a pulse profile by searching for and studying correlations between the measured phases of the harmonic components of pulses or the variance of these phases and the pulsar's X-ray flux or spectrum. The phase residuals of the first and second harmonic components of the pulses of XTE J1814-338 appear to be anticorrelated with its X-ray flux (Chung et al. 2008; Papitto et al. 2007), making it a good candidate for this kind of study. Riggio et al. (2008) have reported a strong anticorrelation between the X-ray flux of XTE J1807-294 and the phase residual of the second harmonic component of its pulse profile. Methods for testing this model of phase variations on timescales shorter than the time required to construct a pulse profile are discussed in Section 4.5.

\subsection{Correlated Pulse Amplitude and Phase Variations}

If the emitting areas of the AMXPs are near their spin axes and move around with time, the amplitudes and phases of their pulses should show two types of correlated behavior.

A strong expectation in this model is that the scatter of the pulse arrival times (i.e., the scatter of the time or phase residuals) should decrease steeply with increasing pulse amplitude. The reason for this is that, as shown previously, a given variation in the azimuthal position of the emitting area produces a much larger phase shift when the emitting area is very close to the 


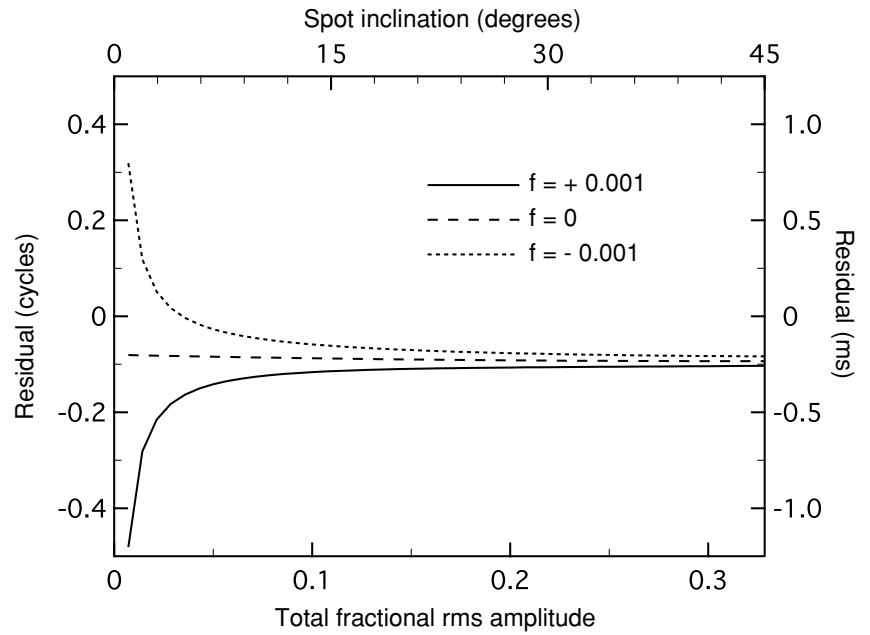

Figure 6. Distribution of pulse arrival times as a function of the pulse amplitude expected for pulses produced by an emitting area that is close to the star's spin axis and wanders. In this example, the emitting area is assumed to wander in stellar longitude by the same distance $d \ell$ at all latitudes. The dotted and solid curves show the expected scatter of pulse arrival times (right vertical axis) and arrival phases (left vertical axis) as a function of the pulse amplitude, assuming $f \equiv d \ell / 2 \pi R= \pm 0.001$. The dashed curve shows the relation between the arrival time and amplitude of pulses produced by a spot that remains at the same longitude as its latitude changes. Here the arrival time of a pulse is taken to be the arrival time of its peak. The phase and time residuals are defined in Section 2.3. These results are for a spot with a radius of $25^{\circ}$ on a $1.4 M_{\odot}$ star with a radius of $5 \mathrm{M}$ spinning at $400 \mathrm{~Hz}$, observed at an inclination of $45^{\circ}$. The amplitude range plotted on the horizontal axis corresponds to spot inclinations from $2^{\circ}$ to $45^{\circ}$ (compare Figure 4 ). This figure illustrates the large scatter expected when the fractional amplitude is small.

spin axis than when it is farther away; in contrast, the oscillation amplitude is much smaller when the emitting area is very close to the spin axis than when it is farther away.

This effect is illustrated in Figure 6, which shows the expected distribution of pulses in the arrival time versus amplitude plane if they are produced by emission from an area close to the star's spin axis that wanders over time. In the example shown, the phase residuals vary by $\sim 0.3$ cycles when the fractional amplitude is $\sim 0.02$ but by only $\sim 0.03$ cycles when the fractional amplitude is $\sim 0.1$. The pulse phase residuals seen in several AMXPs (see, e.g., XTE J1807-294: Patruno 2008; SAX J1748.9-2021: Patruno 2008; and IGR J00291+5934: Patruno 2008) have distributions similar to that shown in Figure 6. These distributions are consistent with the model discussed here if the emitting areas of these AMXPs wander in the azimuthal direction by distances equal to $\sim 10^{-2}-10^{-3}$ of the stellar circumference.

A second expectation in the moving spot model discussed here is that the pulse arrival time or phase residuals are likely to form a track in the phase-residual versus pulse-amplitude plane, especially if the change in the pulse amplitude is large. Such a track will be formed if the emitting areas where the accreting matter impacts the stellar surface tend to move along a favored path in stellar latitude and longitude as the accretion rate and the structure of the inner disk change. This is expected because models of the flow of accreting matter from the inner disk to the stellar surface predict that matter will be guided along particular but different magnetic flux tubes as the accretion rate and the structure of the inner disk vary (see Section 2.1).

Our computations show that motion of the emitting area along a path that changes its latitude but not its longitude can shift the phases of the first and second harmonics by at least 0.15 cycles and by different amounts. Motion of the emitting area along a path that instead changes its longitude but not its latitude can shift the phases of the first and second harmonics and the pulse peak by much larger amounts, if the emitting area is near the spin axis, but in this case the phase shifts will be the same for all Fourier components. Only if the shift in pulse phase produced by the change in the longitude of the emitting area exactly compensates for the shift produced by the change in the latitude of the emitting area will there be no trend in the pulse phase residuals with increasing pulse amplitude.

No track may be discernible in the phase-residual versus pulse-amplitude plane when the pulse amplitude is small because, as has just been discussed, the pulse phase is very sensitive to the position of the emitting area when the area is near the spin axis, which is where it is expected to be when the pulse amplitude is small. If, however, the emitting area moves well away from the spin axis, which in the moving spot model produces a substantial increase in the pulse amplitude, a track may become apparent. Tracks are observed in the data on several AMXPs when the pulse amplitude is $\gtrsim 3 \%$ (Patruno 2008). The phase of the first harmonic component of the pulse is correlated with the amplitude of the pulse in IGR J00291+5934 and XTE J1751-305 but anticorrelated in XTE J1807-294 and XTE J1814-338.

The path on the stellar surface along which the emitting area moves as the accretion rate changes depends on the details of the accretion flow from the inner disk to the stellar surface that cannot yet be determined from first principles. However, the path along which the emitting area moves in the moving spot model can be inferred from simultaneous measurements of the phases and amplitudes of pulses.

Similar shifts in the phases of all Fourier components are consistent with movement of an emitting area of roughly fixed size, shape, and radiation-beaming pattern along a path that causes a substantial change in its longitude as its latitude decreases. This behavior is observed in XTE J1814-338 (Papitto et al. 2007; Chung et al. 2008; Patruno 2008). If instead the phase residuals of different Fourier components of the pulse evolve very differently with increasing pulse amplitude, this is an indication that the emitting area is moving along a path that produces very little change in the area's longitude and/or a substantial change in its shape or the radiation-beaming pattern. This behavior is observed in XTE J1807-294 (Patruno 2008).

\subsection{Accretion- and Nuclear-powered Oscillations}

The close agreement of the pulse profiles and phases of the accretion- and nuclear-powered (X-ray burst) oscillations observed in SAX J1808.4-3658 (Chakrabarty et al. 2003) and XTE J1814-338 (Strohmayer et al. 2003) strongly suggests that in these stars, both types of oscillation are produced by X-ray emission from nearly the same area on the stellar surface. If this is so, it implies that in these AMXPs thermonuclear burning is concentrated near the magnetic poles onto which accreting matter is falling. It also implies that long-term variations in the phase residuals of the two types of oscillations should track one another in these pulsars and should also be correlated with variations in the X-ray flux and spectrum, because both types of variations are produced by changes in the accretion flow through the inner disk.

This interpretation can be tested by comparing the observed variations of the amplitudes and phases of the Fourier components of the accretion- and nuclear-powered oscillations with the variations predicted by this model. If this interpretation proves 
correct, the locations and movements of the emitting areas can be determined from the observed phase and amplitude variations.

In Section 5.1, we emphasize that a mechanism that drives a star's magnetic poles toward its spin axis can greatly reduce the dipole component of the star's magnetic field without reducing significantly its strength. The final configuration of the magnetic field produced by this mechanism could have a total strength $\sim 10^{11}-10^{12} \mathrm{G}$, but a dipole component $\sim 10^{8} \mathrm{G}$, consistent with the dipole moments inferred from the X-ray emission and spin evolution of AMXPs and millisecond radio pulsars (Lamb \& Boutloukos 2008).

Surface magnetic fields $\sim 10^{11}-10^{12} \mathrm{G}$ are strong enough to laterally confine accreting matter within the surface layers of the neutron star, creating an accumulation point for fuel for thermonuclear bursts and causing thermonuclear burning to be concentrated near the star's magnetic poles (Woosley \& Wallace 1982; see also Melatos \& Phinney 2001 and references therein). They are also strong enough to produce strong-magnetic-field features in the keV X-ray spectra of AMXPs (see Mészáros 1992). If this picture is correct, such features are more likely to be detected in AMXPs such as SAX J1808.4-3658 and XTE J1814-338 that produce nuclear-powered oscillations that are phase-synchronized (or nearly synchronized) with their accretion-powered oscillations than in other AMXPs.

\subsection{Effects of Rapid Spot Movements}

Our results show that motion of the emitting area on the stellar surface generally changes both the amplitudes and the phases of the Fourier components of the pulse profile. As noted in Section 2.1, the position of the emitting area is expected to reflect the accretion rate and structure of the inner disk, and is therefore expected to change on timescales at least as short as the $\sim 0.1 \mathrm{~ms}$ dynamical time near the neutron star. Pulse phase and shape variations are therefore expected on this timescale.

The observable effects of changes in the position of the emitting area on timescales longer than the $\sim 10^{2}-10^{3} \mathrm{~s}$ integration times required to construct a pulse profile have been discussed in previous sections. Here we discuss the expected effects of fluctuations in the position of the emitting area on timescales shorter than the time required to construct a pulse profile.

Changes in the position of the emitting area on timescales shorter than the time required to construct a pulse profile will appear as unresolved amplitude and phase noise (see Lamb et al. 1985). This noise will reduce the apparent fractional amplitudes of the oscillations at the spin frequency and its harmonics, making them appear weaker than in the plots presented in this paper, which assume stable emitting spots fixed on the stellar surface. These pulse phase and amplitude fluctuations are likely to be greater when the emitting area is near the spin axis, because there a displacement by a given distance produces a larger change in the pulse phase and, for many geometries, in the pulse amplitude. This trend will tend to reduce further the apparent pulse amplitude when the emitting area is close to the spin axis, making it appear even smaller relative to the pulse amplitude when the emitting area is further from the spin axis than is shown in the plots presented earlier in this section.

Pulse shape fluctuations caused by rapid changes in the position of the emitting region will appear as background noise in excess of the Poisson counting noise. This noise can in principle be detected, especially because its strength is expected to be anticorrelated with the pulse amplitude and to depend in a systematic way on the X-ray flux and spectrum of the pulsar. Detection of more excess noise when the apparent pulse amplitude is smaller would support the moving spot model of pulse phase and amplitude variations.

\subsection{Undetected and Intermittent Pulsations}

The results presented in Section 3 show that if the emitting area is very close to the spin axis and remains there, the amplitude of X-ray oscillations at the stellar spin frequency or its overtones may be so low that they are undetectable. In addition, rapid variations in the shape and phase of pulses are expected to be stronger when the emitting area is very close to the spin axis. The noise produced by these fluctuations mayin combination with other effects, such as reduction of the modulation fraction by scattering in circumstellar gas-further reduce the detectability of accretion-powered oscillations in neutron stars with millisecond spin periods (Lamb et al. 1985; Miller 2000). Taken together, these effects may help explain the nondetection of accretion-powered oscillations in some accreting neutron stars in which nuclear-powered oscillations have been detected.

Location of the X-ray-emitting areas close to the spin axis also suggests a natural explanation for the sudden appearance and disappearance of accretion-powered oscillations observed in the "intermittent" AMXPs (see Lamb et al. 2008; Boutloukos et al. 2008). A change in the accretion flow within the magnetosphere can suddenly channel gas to the stellar surface farther from the spin axis, causing the centroid of the emitting area to move away from the axis. This will increase the amplitude of the oscillation, potentially making a previously undetectable oscillation detectable. Conversely, a change in the accretion flow that suddenly channels accreting gas closer to the spin axis could make a detectable oscillation undetectable. As noted by Lamb et al. (2008), this idea can be tested by studying shortterm variations in the amplitudes and phases of the harmonic components of the pulses.

\section{DISCUSSION}

The results presented in previous sections show that a model of AMXPs in which the X-ray-emitting areas are close to the spin axis and move around on the stellar surface can explain many of their properties. Emitting areas close to the spin axis are to be expected if the magnetic poles of the AMXPs are close to their spin axes, causing accreting gas to be channeled there. In this section, we first discuss mechanisms that may cause the magnetic poles of AMXPs to be close to their spin axes. We then point out that this picture of magnetic field evolution may also explain why AMXPs in which accretion-powered oscillations have been detected are transient X-ray sources. We also discuss several observational tests of this model.

\subsection{Movement of Magnetic Poles Toward the Spin Axis}

The neutron vortices in the fluid core of a spinning neutron star are expected to move radially inward if the star is spun up. This inward vortex motion is expected to drag the magnetic flux tubes in the fluid core of the star toward the star's spin axis (Srinivasan et al. 1990; Ruderman 1991). ${ }^{7}$ Some of the relevant physics is not well understood and many details remain to be explored, but this process is expected to squeeze the magnetic

\footnotetext{
7 It has also been argued (Srinivasan et al. 1990) that a neutron star's magnetic field will be greatly reduced earlier in its evolution if it is spun down by a large factor, causing outward moving neutron vortices to force its magnetic field into the crust near the rotation equator, where it can be dissipated.
} 
flux of accreting pulsars toward their spin axes as they are spun up to short periods (recycled). As a result, the magnetic poles of recycled millisecond pulsars are expected to be very close to their spin axes (Chen \& Ruderman 1993; Chen et al. 1998). The magnetic poles of recycled pulsars could also end up close to their spin axes if the accretion spin-up torque has a component that tends to align the star's magnetic field with its spin axis as it spins up. Motion of the magnetic poles toward the spin axis will be facilitated by diffusion of magnetic flux through the crust and/or fracture and fragmentation of the crust (Ruderman 1991).

If the star's north and south magnetic poles are in opposite rotation hemispheres when spin-up begins, the inward motion of vortices will drag them toward opposite spin poles. If instead both poles are in the same rotation hemisphere when spin-up begins, they will be dragged toward the same spin pole. In either case, the strength of the magnetic field at the stellar surface will be orders of magnitude larger than the strength of its dipole component, as we now explain.

An initial magnetic field $M_{1}$ that is approximately uniform over a stellar hemisphere of radius $R$ has a magnetic moment $\mu_{1} \approx M_{1} R^{3}$. The magnetic flux threading the fluid core is conserved as the magnetic field is squeezed. Suppose the inward moving vortices squeeze the flux into a tube of radius $a \approx R / 30$. This will produce a squeezed magnetic field of strength $M_{2} \approx(R / a)^{2} M_{1} \approx 10^{3} M_{1}$.

If the star's north and south magnetic poles are forced toward opposite spin poles, the dipole moment of the squeezed magnetic field will be aligned with the star's spin axis and will have a magnitude $\mu_{2} \approx M_{2} R a^{2} \approx \mu_{1}$, corresponding to a surface magnetic field with a dipole component $M_{2}$ (dipole) $\approx M_{1}$. The full strength $M_{2}$ of the surface magnetic field will therefore be $\approx(R / a)^{2} \approx 10^{3}$ times larger than the strength $M_{2}$ (dipole) of its dipole component.

If instead the star's north and south magnetic poles are both forced toward the same spin pole, the dipole moment of the squeezed magnetic field will be orthogonal to the spin axis and hence the star will be an "orthogonal rotator," even though both magnetic poles are near a spin pole rather than near the spin equator. In this case the dipole moment will have a magnitude $\mu_{2} \approx M_{2} L a^{2} \approx(L / R) \mu_{1}$, where $L$ is the final separation between the north and south magnetic poles, corresponding to a surface magnetic field with a dipole component $M_{2}($ dipole $) \approx$ $(L / R) M_{1}$. Thus if $L \approx 0.1 R$, the dipole moment of the squeezed field will be about 10 times smaller than the dipole moment of the original field and the full strength $M_{2}$ of the surface magnetic field will be $\approx(R / L)(R / a)^{2} \approx 10^{4}$ times larger than the strength $M_{2}$ (dipole) of its dipole component.

Regardless of whether the star's north and south magnetic poles are forced toward opposite spin poles, creating an aligned rotator, or toward the same spin pole, creating an orthogonal rotator, both magnetic poles will end up very close to the spin axis. Accreting gas that is channeled along magnetic field lines will therefore impact the stellar surface close to the spin axis, and accretion-powered X-ray emission will come primarily from regions near the spin axis.

Note that if a pulsar's north and south magnetic poles have both been forced close to the same spin axis, the flow of accreting matter could impact the surface in a pattern with predominantly either onefold or twofold symmetry relative to the spin axis. In this accretion geometry, even a small change in the properties of the flow through the inner disk could change the dominant symmetry of the emitting area from onefold to twofold or vice versa, thereby changing the relative strengths of the first and second harmonics in the pulse profile.

Another consequence of this picture of magnetic field evolution is that both accretion-powered (X-ray) and rotationpowered (radio) millisecond pulsars may have surface magnetic fields $\sim 10^{11}-10^{12} \mathrm{G}$. This is $\sim 10^{3}-10^{4}$ times stronger than the $\sim 10^{8}-10^{9} \mathrm{G}$ dipole components inferred from the magnetospheric radii of AMXPs (see, e.g., Psaltis \& Chakrabarty 1999; Lamb \& Boutloukos 2008), the spin-down of SAX J1808.4-3658 in quiescence (Hartman et al. 2008), and the spin-down rates of the rotation-powered millisecond radio pulsars (see Lamb \& Boutloukos 2008). Magnetic fields this strong are strong enough to create an accumulation point for fuel for thermonuclear bursts and produce strong-magnetic-field features in the keV X-ray spectra of AMXPs (see Section 4.4).

\subsection{Why AMXPs are Transients}

The picture of the X-ray emission and magnetic field evolution of AMXPs that we have outlined here suggests a possible explanation for why all AMXPs found so far are transients. These systems have very low long-term average mass transfer rates, but binary modeling suggests that the mass transfer rates were higher in the past (see, e.g., Bildsten \& Chakrabarty 2001 for a discussion in the context of SAX J1808.4-3658). If stars such as these are initially spun up to high spin rates, so that their magnetic poles are forced very close to their spin axes, they would appear similar to the accreting neutron stars in low-mass $\mathrm{X}$-ray binary systems in which accretion-powered oscillations have not been detected. When their accretion rates later decrease, and magnetic dipole and other braking torques cause them to spin down, their magnetic poles will be forced away from the rotation axis, and their accretion-powered oscillations will become detectable.

If this explanation of the transient nature of the AMXPs is correct, they should be spinning down on long timescales, as seems to be the case (see, e.g., Hartman et al. 2008), and the amplitudes and phases the harmonic components of their waveforms should evolve in a correlated way as their magnetic poles move away from their spin axes (see Section 4).

\subsection{Comparison with the Properties of MRPs}

Rotation-powered millisecond radio pulsars (MRPs) are thought to be the offspring of AMXPs and therefore should have magnetic field geometries similar to those of the AMXPs, except that the low rate of accretion at the end of the accretion phase and magnetic dipole braking toward the end of the accretion phase and afterward will tend to spin them back down, forcing their magnetic poles away from their spin axes.

Although the radio emission properties of most MRPs provide little clear evidence about the locations of their magnetic poles (see Manchester \& Han 2004), there are indications from their radio emission that the dipole moments of a substantial number of the most rapidly spinning MRPs in the galactic disk are nearly aligned or nearly orthogonal to their spin axes (Chen et al. 1998; see also Chen \& Ruderman 1993). As explained above, either orientation is consistent with their magnetic poles being driven close to their spin axes by neutron vortex motion during spin-up as AMXPs.

Analysis and modeling of the waveforms of the thermal Xray emission from MRPs may provide better constraints on their magnetic field geometries. Recent modeling of the high (30\% to 
$50 \%$ rms) amplitude X-ray oscillations observed in three nearby MRPs using Comptonized emission and two antipodal or nearly antipodal hot spots is consistent with their emitting regions being far from their spin axes (Bogdanov et al. 2007, 2008). We note that all three pulsars have relatively low $(\sim 150 \mathrm{~Hz}-200 \mathrm{~Hz})$ spin rates and may therefore have been spun down by a factor $\sim 3$ from their maximum spin frequencies after spin-up. If so, their magnetic poles may have been forced away from their spin axes by a similar factor. This could allow consistency between the $\sim 40^{\circ}-60^{\circ}$ inclinations inferred by Bogdanov et al. for these three MRPs and the $\lesssim 20^{\circ}$ inclinations typically required in the model of AMXPs described here. We also note that only MRPs that produce high-amplitude X-ray oscillations are currently detectable as oscillators, so the ones that are detected may have magnetic inclinations larger than is typical. More investigation is needed.

\subsection{Other Observational Tests}

In the nearly aligned moving spot model of AMXP X-ray emission, the properties of X-ray pulses (e.g., their amplitudes, harmonic content, and arrival times) should be functions of the pulsar's X-ray luminosity and spectrum. The reason is that the properties of pulses are determined by the location of the emitting area, which depends on the rate and structure of the accretion flow through the inner disk. The X-ray luminosity and spectrum of the pulsar will also depend on the accretion flow through the inner disk. Hence the properties of pulses should be correlated with the X-ray luminosity and spectrum.

Changes in the location of the emitting area on the stellar surface on timescales longer than the $\sim 10^{2}-10^{3} \mathrm{~s}$ intervals required to construct stable pulse profiles will produce correlated changes in the amplitudes, harmonic content, and arrival times of these profiles.

In the nearly aligned moving spot model, the arrival times of pulses with low amplitudes are expected to fluctuate much more than the arrival times of pulses with high amplitudes (see Figure 6). The reason is that pulse amplitudes are lower when the emitting area is closer to the spin axis, where small variations in the position of the emitting area can produce large variations in the pulse arrival time.

A second expectation is that pulse phase residuals will form a track in the phase-residual versus pulse-amplitude plane. This will be the case if the area where accreting matter impacts the stellar surface and X-rays are emitted tends to move along a favored path on the stellar surface as the accretion flow through the inner disk changes. The location of the emitting area on its favored path will depend on the accretion flow through the inner disk and will in turn determine the position of pulses in the phase-residual versus pulse-amplitude plane. Changes in the accretion flow through the inner disk will therefore cause correlated changes in the phases and amplitudes of pulses, producing a track when these are plotted in the phase-residual versus pulse-amplitude plane. Such tracks are likely to be more evident if the range of the pulse-amplitude variation is large.

The pulses of IGR J00291+5934, XTE J1751-305, XTE J1807-294, and XTE J1814-338 seem to form track-like patterns in the phase-residual versus pulse-amplitude plane when the pulse amplitude is $\gtrsim 5 \%$ (see Section 4.3). An important expectation of the moving spot model is that the position of pulses along such a track should be correlated with the X-ray luminosity and spectrum of the pulsar.

Another check of the moving spot model is possible if the nearly identical pulse profiles and phases of the accretion- and nuclear-powered X-ray oscillations of XTE J1814-338 and SAX J1808.4-3658 are produced by emission from the same area on the stellar surface, as suggested in Section 4.4. If this is the case, the model predicts that longer-term (days-weeks) variations of the phase residuals of the accretion- and nuclearpowered oscillations should be correlated with one another and with longer term variations of the pulsar's X-ray luminosity and spectrum, because all three variations are expected to be related to changes in the accretion flow through the inner disk. Evidence of this behavior would support the moving spot model.

The location of the emitting area on the stellar surface is likely to change on timescales as short as the $\sim 1 \mathrm{~ms}$ dynamical timescale in the inner disk. These rapid movements of the emitting area will cause rapid variations of the observed Xray flux. Flux variations on timescales shorter than the $\sim 10^{2}-$ $10^{3} \mathrm{~s}$ intervals required to construct stable pulse profiles are observable as noise in excess of the photon counting noise. In the moving spot model, the motion of the emitting area that produces this excess noise also affects the amplitudes, harmonic content, and arrival times of the X-ray pulses, and is related to the X-ray luminosity and spectrum. Hence the strength of the excess noise produced by motion of the emitting area should be correlated with these other properties of the AMXP.

A strong expectation in the nearly aligned moving spot model is that the component of the excess noise produced by motion of the emitting area should be stronger when the pulse amplitude is smaller and weaker when the pulse amplitude is larger (see Section 4.3).

One way to focus on the strength of the noise produced by movement of the emitting area would be to filter the X-ray flux data to remove variations on timescales shorter than, say, $0.05 \mathrm{~s}$. This would remove the variability associated with oscillations at the pulsar spin frequency and any high-frequency QPOs. The remaining total variability would then be correlated with the other properties of the AMXP listed above. Detection of greater variability when the observed pulse amplitude is smaller would support the nearly aligned moving spot model.

If AMXPs do have surface magnetic fields as strong as $\sim 10^{11}-10^{12} \mathrm{G}$, as suggested in Section 5.1, their keV spectra may show strong-magnetic-field features. Such features may include increased flux below the fundamental cyclotron resonance energy, where the magnetic field suppresses the opacity of surface layers (see, e.g., Pavlov et al. 1975); resonance scattering features produced by the fundamental cyclotron resonance and its overtones (see, e.g., Mészáros \& Nagel 1985); and changes in the spectrum at the higher energies where the cyclotron scattering becomes unimportant.

Another implication of the existence of AMXPs with surface magnetic fields as strong as $\sim 10^{11}-10^{12} \mathrm{G}$ is that the rotationpowered millisecond pulsars that are their progeny should have surface magnetic fields this strong, even though their dipole fields are $\sim 10^{8}-10^{9} \mathrm{G}$. Magnetic fields this strong can be expected to affect particle acceleration and $\gamma$-ray emission by these pulsars.

\section{CONCLUSIONS}

In previous sections, we have explored in some detail the nearly aligned moving spot model of AMXP X-ray emission. In this model, the X-ray-emitting regions are close to the stellar spin axis and move around on the stellar surface with time. Here we list our principal conclusions. 
Pulse amplitudes and shapes. In Section 3.1, we investigated the amplitudes and shapes of the pulses produced by emitting spots on the stellar surface as a function of their inclination to the spin axis, for several X-ray-beaming patterns and a range of stellar masses, compactnesses, and spin rates. We found that emitting areas on or near the stellar surface can produce fractional amplitudes as low as the $1 \%-2 \%$ values often observed only if they are located within a few degrees of the stellar spin axis. Regions near the spin axis also naturally produce nearly sinusoidal pulse profiles.

We explored effect of spot size on pulse amplitude in Section 3.2. We found that although the pulse amplitudes produced by large emitting areas tend to be smaller, this effect is weak. Unless almost the entire surface of the star is uniformly emitting, even large spots produce pulse amplitudes greater than those observed in the AMXPs, unless the spots are centered close to the spin axis.

In Section 3.3, we studied the effect of stellar compactness on pulse amplitudes. Although the pulse amplitudes produced by very compact neutron stars tend to be smaller than the amplitudes produced by less compact stars, we found that this effect is too weak to explain by itself pulse amplitudes as small as those observed in the AMXPs. Stellar compactness clearly cannot explain why the fractional amplitudes of several AMXPs are $\sim 1 \%-2 \%$ at some times but $\sim 15 \%-25 \%$ a few hours or days later, because the stellar compactness cannot change on such short timescales.

These results show that emission from the stellar surface can explain the low amplitudes and nearly sinusoidal waveforms typically observed in AMXPs only if the emitting areas are located close to the stellar spin axis.

Variability of pulse amplitudes, shapes, and arrival times. In Section 4.1, we investigated the amplitude changes that can be produced by motion of the emitting area on the stellar surface. We found that if the emitting area is close to the spin axis, even a small change in the latitude of the area can change the oscillation amplitude by a substantial factor. For example, changes in the inclination of the emitting area by $\lesssim 10^{\circ}$ can explain the amplitude variations seen in the AMXPs and the relatively large fractional amplitudes $\sim 15 \%-20 \%$ occasionally seen in some of them.

In Section 4.2, we studied the changes in the arrival times (phases) of the harmonic components of the pulse caused when the emitting area moves around on the stellar surface. We found that changes in the latitude of the emitting area can shift the phases of the first and second harmonics by at least 0.15 cycles and by different amounts. We showed that if the emitting area is close to the spin axis and moves in the azimuthal direction by even a small distance, the phases of the first and second harmonics can easily shift by as much as $\sim 0.1-0.4$ cycles. If the emitting area loops the spin axis, the phases of the Fourier components will shift by more than one cycle.

Motion of the emitting area on the stellar surface generally produces both amplitude and phase variations. As discussed in Section 2, the position of the emitting area is expected to reflect the accretion rate and structure of the inner disk, and is therefore expected to change on timescales at least as short as the $\sim 0.1 \mathrm{~ms}$ dynamical time at the stellar surface and as long as the $\sim 10$ day timescale of the variations observed in the mass accretion rate. Changes in the position of the emitting area on timescales longer than the $\sim 10^{2}-10^{3}$ s integration times required to construct a pulse profile will produce changes in the apparent pulse amplitude and phase.
These results show that if the emitting area is close to the spin axis, modest changes in its location can explain the rapidly varying harmonic amplitudes and phases of the AMXPs.

Correlated amplitude and phase variations. We showed in Section 4.3 that changes in the latitude and longitude of the emitting area tend to produce correlated changes in the amplitudes and phases of the harmonic components of the pulse. A strong expectation in the nearly aligned moving spot model is that the scatter in the pulse arrival times (i.e., the pulse time or phase residuals) should decrease steeply with increasing pulse amplitude. The residuals of several AMXPs, including XTE J1807-294, SAX J1748.9-2021, and IGR J00291+5934, behave in this way. The magnitudes of the phase residuals of these AMXPs are consistent with the nearly aligned moving spot model if the emitting areas wander in the azimuthal direction by distances $\sim 10^{-2}-10^{-3}$ times the stellar circumference.

A second expectation in the nearly aligned moving spot model is that the arrival times of pulses will form a track in the phaseresidual versus pulse-amplitude plane, especially if the change in the pulse amplitude is large. Such a track will be formed if the emitting areas where the accreting matter impacts the stellar surface move repeatedly along a particular path in stellar latitude and longitude as the accretion rate and the structure of the inner disk change. This is expected because models of the flow of accreting matter from the inner disk to the stellar surface predict that matter will be guided along particular but different magnetic flux tubes as the accretion rate and the structure of the inner disk vary (see Section 2.1). As discussed in Section 4.3, tracks of this type are observed in plots of pulse arrival time versus pulse amplitude for XTE J1814-338 and XTE J1807-294.

Accretion- and nuclear-powered oscillations. The success of the emitting spot model discussed here supports magnetic field evolution models in which the magnetic flux of the accreting neutron star becomes concentrated near its spin axis as it is spun up. As discussed in Sections 4.4 and 5.1, these evolutionary models can produce magnetic fields strong enough to partially confine accreting nuclear fuel near the star's magnetic poles, even though the dipole component of the magnetic field is very weak. This picture of magnetic field evolution in turn suggests that the shapes and phases of the nuclear- and accretion-powered pulses are similar to one another in some AMXPs because the nuclear- and accretion-powered X-ray emission comes from approximately the same area on the stellar surface.

Effects of rapid spot motions. In Section 4.5, we discussed the effects of spot movements on timescales shorter than the time required to construct a pulse profile. Such effects are expected, because emitting spots are likely to move on the stellar surface on timescales at least as short as the $\sim 0.1 \mathrm{~ms}$ dynamical time there whereas constructing a pulse profile usually requires folding $\sim 10^{2}-10^{3} \mathrm{~s}$ of X-ray flux data. Rapid spot motions will produce X-ray flux variations on these same timescales. Our computations show that motion of the emitting area on the stellar surface on timescales longer than the spin period usually changes the amplitudes and the phases of the harmonic components of the theoretical pulse profile.

Variations of the X-ray flux on any timescales shorter than the time required to construct a pulse profile will appear in the analysis as noise in excess of the normal counting noise. This noise will reduce the measured amplitude of the oscillations at the spin frequency and its overtones for all spot locations, but its effect is likely to be stronger when the emitting area is near the spin axis because displacement of the emitting area by a given distance there produces a larger change in the pulse phase 
and, for many geometries, in the pulse amplitude. This effect will therefore tend to reduce the apparent pulse amplitude even further when the emitting area is close to the spin axis.

Undetectable and intermittent pulsations. In Section 3.1, we showed that if the emitting areas of some AMXPs are very close to the spin axis and remain there, the amplitudes of the oscillations they would produce can be $\sim 0.5 \%$ or less, making them undetectable with current instruments. Rapid X-ray flux variations will make accretion-powered oscillations at the spin frequency more difficult to detect. Other effects, such as scattering of X-ray photons in circumstellar gas, may also play a role in reducing the detectability of such oscillations. These results show that the nearly aligned moving spot model may, possibly in combination with other effects, explain the nondetection of accretion-powered oscillations at the millisecond spin periods of some accreting neutron stars in which nuclear-powered oscillations have been detected. In Section 4.1, we showed that if the emitting area is within a few degrees of the spin axis and moves toward the rotation equator by $\sim 10^{\circ}$, oscillations that were undetectable can become detectable. This may explain why accretion-powered oscillations appear only intermittently in some AMXPs (Lamb \& Boutloukos 2008). The model suggests that oscillations may also disappear intermittently in some AMXPs.

Evolution of AMXP magnetic fields. In Section 5.1, we explained why the magnetic poles of most AMXPs are expected to be very close to their spin axes. One consequence is that their magnetic fields will channel accreting gas to the stellar surface near the spin axis. Hence the star's accretion-powered $\mathrm{X}$-ray emission will come from areas near the spin poles. A second consequence is that many AMXPs may have surface magnetic fields as strong as $\sim 10^{11}-10^{12} \mathrm{G}$, even though the dipole components of these fields are only $\sim 10^{8}-10^{9} \mathrm{G}$. If so, many MRPs may also have surface magnetic fields as strong as $\sim 10^{11}-10^{12} \mathrm{G}$, even though the dipole components inferred from their spin-down rates are only $\sim 10^{8}-10^{9} \mathrm{G}$.

Transient nature of AMXPs. In Section 5.2, we noted that the picture of AMXP magnetic field evolution just described suggests why the AMXPs in which accretion-powered oscillations have been detected are in transient systems. If the magnetic poles of most neutron stars in LMXBs were forced very close to their spin axes during the initial, persistent phase of mass transfer, accretion-powered X-ray oscillations would be difficult or impossible to detect. Later, when mass transfer is transient, the stars will spin down, causing their magnetic poles to move away from their spin axes and making accretion-powered X-ray oscillations detectable.

Tests of the model. The nearly aligned moving spot model leads to a number of expectations about AMXP X-ray emission that can be tested (see Section 5 for details):

1. The amplitudes, harmonic content, and arrival times of pulses should be functions of the X-ray luminosity and spectrum of the pulsar.

2. The amplitudes, harmonic content, and arrival times of pulses should be correlated with one another.

3. The arrival times of pulses with low amplitudes should fluctuate much more than the arrival times of pulses with high amplitudes.

4. Pulse phase residuals are likely to form a track in the phase-residual versus pulse-amplitude plane. Such tracks are likely to be more evident if the range of the pulse amplitude variation is large. The position of pulses along such a track should be correlated with the X-ray luminosity and spectrum of the pulsar.

5. If the accretion- and nuclear-powered pulses of an AMXP appear nearly identical, long-term (days-to-weeks) variations in the phase residuals of the two types of pulses should track one another.

6. The strength of the excess background noise produced by pulse shape fluctuations should be correlated with the amplitudes, harmonic content, and arrival times of pulses and the X-ray luminosity and spectrum of the pulsar.

7. The excess noise produced by motion of the emitting area should be stronger when the pulse amplitude is smaller and weaker when the pulse amplitude is larger.

8. If AMXPs do have surface magnetic fields as strong as $\sim 10^{11}-10^{12} \mathrm{G}$, their keV X-ray spectra may show strongmagnetic-field features. Such features are more likely in AMXPs that show evidence of nuclear fuel confinement, such as SAX J1808.4-3658 and XTE J1814-338.

9. If AMXPs have surface magnetic fields $\sim 10^{11}-10^{12} \mathrm{G}$, their offspring should have millisecond spin periods and total magnetic fields of similar strength, but dipole fields $\sim 10^{8}-10^{9} \mathrm{G}$. This should affect particle acceleration and $\gamma$-ray emission by these neutron stars.

10. If the explanation of the transient nature of the AMXPs suggested here is correct, they should be spinning down on long timescales.

Note added in manuscript: This work was presented at the 2008 April Amsterdam Workshop on Accreting Millisecond Pulsars, where we emphasized a test of our nearly aligned moving spot model that could also have wider implications. We argued that the close similarity of the accretion-powered and burst oscillation waveforms in XTE J1814-338 strongly suggests that the emitting regions that produce them are similar and collocated, and that if, as we had proposed previously, the phase wandering of the accretion-powered oscillations is caused by movement of the emitting region, then the phase of the burst oscillations should wander in the same way. After the Workshop, Watts et al. (2008) investigated this possibility and found just such a correlation in the RXTE data on XTE J1814-338. In particular, these authors found that during the main part of the two-month outburst of XTE J1814-338, its burst oscillation not only has a waveform similar to that of the accretion-powered oscillation (Strohmayer et al. 2003; Watts et al. 2005, 2006), but is also phase-locked with it, and that the peak of the burst oscillation coincides with the peak of the soft component of the accretion-powered oscillation. This indicates that, as we had suggested, the accretion- and nuclear-powered emitting regions in this pulsar very nearly coincide, and that the simultaneous wandering of the arrival times of both oscillations by $\sim 1 \mathrm{~ms}$ $(\sim 0.3$ in phase) during the outburst is due to wandering of the matter (and hence the fuel) deposition pattern on the stellar surface.

We thank Deepto Chakrabarty, Paul Demorest, Jacob Hartman, Mike Muno, Alessandro Patruno, Scott Ransom, Ingrid Stairs, Michiel van der Klis, and Anna Watts for helpful discussions. We thank Anthony Chan and Aaron Hanks for assistance in analyzing and plotting our results. The results presented here are based on research supported by NASA grant NAG 5-12030, NSF grant AST0709015, and funds of the Fortner Endowed Chair at Illinois, and by NSF grant AST0708424 at Maryland. 


\section{REFERENCES}

Altamirano, D., Casella, P., Patruno, A., Wijnands, R., \& van der Klis, M. 2008, ApJ, 674, L45

Arons, J., \& Lea, S. M. 1976, ApJ, 207, 914

Basko, M. M., \& Sunyaev, R. A. 1975, A\&A, 42, 311

Basko, M. M., \& Sunyaev, R. A. 1976, MNRAS, 175, 395

Bildsten, L., \& Chakrabarty, D. 2001, ApJ, 557, 292

Bogdanov, S., Grindlay, J. E., \& Rybicki, G. B. 2008, ApJ, 689, 407

Bogdanov, S., Rybicki, G. B., \& Grindlay, J. E. 2007, ApJ, 670, 668

Boutloukos, S., \& Lamb, F. K. 2008, in AIP Conf. Proc. 983, 40 Years of Pulsars: Millisecond Pulsars, Magnetars, and More, ed. C. G. Bassa et al. (Melville, NY: AIP), 533

Boutloukos, S., Lamb, F. K., Clare, A., Dorris, D., Van Wassenhove, S., Yu, W., \& Miller, M. C. 2008, BAAS, 40, 24.04

Braje, T., Romani, R., \& Rauch, K. 2000, ApJ, 531, 447

Cadeau, C., Morsink, S. M., Leahy, D., \& Campbell, S. S. 2007, ApJ, 654, 458

Casella, P., Altamirano, D., Patruno, A., Wijnands, R., \& van der Klis, M. 2008, ApJ, 674, L41

Chakrabarty, D. 2005, in ASP Conf. Ser. 328, Binary Radio Pulsars, ed. F. A. Rasio \& I. H. Stairs (San Francisco, CA: ASP), 279

Chakrabarty, D., Morgan, E. H., Muno, M. P., Galloway, D. K., Wijnands, R., van der Klis, M., \& Markwardt, C. B. 2003, Nature, 424, 42

Chandrasekhar, S. 1960, Radiative Transfer (Dover: New York)

Chen, K., \& Ruderman, M. 1993, ApJ, 408, 179

Chen, K., Ruderman, M., \& Zhu, T. 1998, ApJ, 493, 397

Chou, Y., Chung, Y., Hu, C.-P., \& Yang, T.-C. 2008, ApJ, 678, 1316

Chung, C. T. Y., Galloway, D. K., \& Melatos, A. 2008, MNRAS, 391, 2554

Daumerie, P., Kalogera, V., Lamb, F. K., \& Psaltis, D. 1996, Nature, 382, 141

Elsner, R. F., \& Lamb, F. K. 1976, Nature, 262, 356

Elsner, R. F., \& Lamb, F. K. 1977, ApJ, 215, 897

Elsner, R. F., \& Lamb, F. K. 1984, ApJ, 278, 326

Galloway, D. K., Chakrabarty, D., Morgan, E. H., \& Remillard, R. A. 2002, ApJ, 576, L137

Galloway, D. K., Markwardt, C. B., Morgan, E. H., Chakrabarty, D., \& Strohmayer, T. E. 2005, ApJ, 622, L45

Galloway, D. K., Morgan, E. H., \& Chakrabarty, D. 2008, in AIP Conf. Proc. 1068, A Decade of Accreting Millisecond X-ray Pulsars, ed. R. Wijnands et al. (Melville, NY: AIP), 55

Galloway, D., Morgan, E. H., Krauss, M. I., Kaaret, P., \& Chakrabarty, D. 2007, ApJ, 654, L73

Gavriil, F. P., Strohmayer, T. E., Swank, J. H., \& Markwardt, C. B. 2007, ApJ, 669, L29

Ghosh, P., \& Lamb, F. K. 1979a, ApJ, 232, 259

Ghosh, P., \& Lamb, F. K. 1979b, ApJ, 234, 296

Ghosh, P., Lamb, F. K., \& Pethick, C. J. 1977, ApJ, 217, 578

Hartman, J. M., et al. 2008, ApJ, 675, 1468

Krimm, H. A., et al. 2007, ApJ, 668, L147

Kulkarni, A. K., \& Romanova, M. M. 2008, MNRAS, 386, 673

Lamb, F. K. 1975a, in X-rays in Space, ed. D. Venkatesan (Calgary: Univ. of Calgary), 613

Lamb, F. K. 1975b, Ann. New York Acad. Sci., 262, 331

Lamb, F. K. 1977, Ann. New York Acad. Sci., 302, 482

Lamb, F. K. 1989, in Timing Neutron Stars, ed. H. Ögelman \& E. P. J. van den Heuvel (Dordrecht: Kluwer), 649

Lamb, F. K., \& Boutloukos, S. 2008, in Short-Period Binary Stars: Observations, Analyses, and Results, Astrophys. and Space Sci. Library, Vol. 352, ed. E. F. Malone, D. A. Leahy, \& D. W. Hobill (Dordrecht: Springer), 87

Lamb, F. K., Boutloukos, S., Clare, A., Dorris, D., Van Wassenhove, S., Yu, W., \& Miller, M. C. 2007, BAAS, 39, 120.04

Lamb, F. K., Boutloukos, S., Clare, A., Dorris, D., Van Wassenhove, S., Yu, W., \& Miller, M. C. 2008, BAAS, 40, 10.27
Lamb, F. K., Boutloukos, S., Van Wassenhove, S., Chamberlain, R. T., Lo, K. H., \& Miller, M. C. 2009, ApJ, 705, L36

Lamb, F. K., Dorris, D., Clare, A., Van Wassenhove, S., Yu, W., \& Miller, M. C. 2006, BAAS, 38, 4.07

Lamb, F. K., \& Miller, M. C. 2001, ApJ, 554, 1210

Lamb, F. K., Pethick, C. J., \& Pines, D. 1973, ApJ, 184, 271

Lamb, F. K., Shibazaki, N., Alpar, M. A., \& Shaham, J. 1985, Nature, 317, 681

Lattimer, J. M., \& Prakash, M. 2001, ApJ, 550, 426

Liu, Q. Z., van Paradijs, J., \& van den Heuvel, E. P. J. 2007, A\&A, 469, 807

Long, M., Romanova, M. M., \& Lovelace, R. V. E. 2008, MNRAS, 386, 1274

Manchester, R. N., \& Han, J. L. 2004, ApJ, 609, 354

Markwardt, C. B. 2004, in AIP Conf. Proc. 714, X-ray Timing 2003: Rossi and Beyond, ed. P. Kaaret, F. K. Lamb, \& J. H. Swank (Melville, NY: AIP), 217

Markwardt, C. B., Swank, J. H., Strohmayer, T. E., in't Zand, J. J. M., \& Marshall, F. E. 2002, ApJ, 575, L21

McCray, R., \& Lamb, F. K. 1976, ApJ, 204, L115

Melatos, A., \& Phinney, E. S. 2001, PASA, 18, 421

Mészáros, P. 1992, High-Energy Radiation from Magnetized Neutron Stars (Chicago, IL: Univ. of Chicago Press)

Mészáros, P., \& Nagel, W. 1985, ApJ, 299, 138

Michel, F. C. 1977, ApJ, 214, 261

Miller, G. 1996, ApJ, 468, L29

Miller, M. C. 2000, ApJ, 537, 342

Miller, M. C., \& Lamb, F. K. 1998, ApJ, 499, L37

Miller, M. C., Lamb, F. K., \& Psaltis, D. 1998, ApJ, 508, 791

Morgan, E. H., Chakrabarty, D., Wijnands, R., van der Klis, M., \& Marwardt, C. 2003, BAAS, 35,629

Muno, M. P., Özel, F., \& Chakrabarty, D. 2002, ApJ, 581, 550

Page, D. 1995, ApJ, 442, 273

Papitto, A., Di Salvo, T., Burderi, L., Menna, M. T., Lavagetto, G., \& Riggio, A. 2007, MNRAS, 375, 971

Patruno, A. 2008, in AIP Conf. Proc. 1068, A Decade of Accreting Millisecond X-ray Pulsars, ed. R. Wijnands, et al. (Melville, NY: AIP), 25

Pavlov, G. G., \& Kaminker, A. D. 1975, Pis'ma Astron. Zh., 1, 13

Pechenick, K. R., Ftaclas, C., \& Cohen, J. M. 1983, ApJ, 274, 846

Poutanen, J., \& Beloborodov, A. M. 2006, MNRAS, 373, 836

Poutanen, J., \& Gierliński, M. 2003, MNRAS, 343, 1301

Psaltis, D., \& Chakrabarty, D. 1999, ApJ, 521, 332

Riggio, A., Di Salvo, T., Burderi, L., Menna, M. T., Papitto, A., Iaria, R., \& Lavagetto, G. 2008, ApJ, 678, 1273

Romanova, M. M., Kulkarni, A. K., \& Lovelace, R. V. E. 2008, ApJ, 673 L171

Romanova, M. M., Kulkarni, A., Long, M., Lovelace, R. V. E., Wick, J. V., Ustyugova, G. V., \& Koldoba, A. V. 2006, Adv. Space Res., 38, 2887

Romanova, M. M., Ustyugova, G. V., Koldoba, A. V., \& Lovelace, R. V. E. 2004, ApJ, 610, 920

Romanova, M. M., Ustyugova, G. V., Koldoba, A. V., Wick, J. V., \& Lovelace, R. V. E. 2003, ApJ, 595, 1009

Ruderman, M. 1991, ApJ, 366, 261

Srinivasan, G., Bhattacharya, D., Muslimov, A., \& Tsygan, A. 1990, Curr. Sci., 59,31

Strohmayer, T. 1992, ApJ, 388, 138

Strohmayer, T., Markwardt, C. B., Swank, J. H., \& 't Zand, J. 2003, ApJ, 596, L67

Viironen, K., \& Poutanen, J. 2004, A\&A, 426, 985

Watts, A. L., Patruno, A., \& van der Klis, M. 2008, ApJ, 688, L37

Watts, A. L., Strohmayer, T., \& Markwardt, C. B. 2005, ApJ, 634, 547

Watts, A. L., \& Strohmayer, T. 2006, MNRAS, 373, 769

Wijnands, R. 2006, in Trends in Pulsar Research, ed. J. A. Lowry (New York: Nova Science), 53

Woosley, S. E., \& Wallace, R. K. 1982, ApJ, 258, 716

Zhang, F., Qu, J., Zhang, C. M., Chen, W., \& Li, T. P. 2006, ApJ, 646, 1116 